\title{
TU/e ENHOUEN

\section{Control of the tokamak safety factor profile with time-varying constraints using MPC}

\section{Citation for published version (APA):}

Maljaars, E., Felici, F. A. A., Baar, de, M. R., Dongen, van, J., Hogeweij, G. M. D., Geelen, P. J. M., \& Steinbuch, M. (2015). Control of the tokamak safety factor profile with time-varying constraints using MPC. Nuclear Fusion, 55(2), 023001-1/18. [023001]. https://doi.org/10.1088/0029-5515/55/2/023001

DOI:

10.1088/0029-5515/55/2/023001

Document status and date:

Published: 01/01/2015

\section{Document Version:}

Publisher's PDF, also known as Version of Record (includes final page, issue and volume numbers)

\section{Please check the document version of this publication:}

- A submitted manuscript is the version of the article upon submission and before peer-review. There can be important differences between the submitted version and the official published version of record. People interested in the research are advised to contact the author for the final version of the publication, or visit the $\mathrm{DOI}$ to the publisher's website.

- The final author version and the galley proof are versions of the publication after peer review.

- The final published version features the final layout of the paper including the volume, issue and page numbers.

Link to publication

\section{General rights}

Copyright and moral rights for the publications made accessible in the public portal are retained by the authors and/or other copyright owners and it is a condition of accessing publications that users recognise and abide by the legal requirements associated with these rights.

- Users may download and print one copy of any publication from the public portal for the purpose of private study or research.

- You may not further distribute the material or use it for any profit-making activity or commercial gain

- You may freely distribute the URL identifying the publication in the public portal.

If the publication is distributed under the terms of Article 25fa of the Dutch Copyright Act, indicated by the "Taverne" license above, please follow below link for the End User Agreement:

www.tue.nl/taverne

Take down policy

If you believe that this document breaches copyright please contact us at:

openaccess@tue.nl

providing details and we will investigate your claim. 
Control of the tokamak safety factor profile with time-varying constraints using MPC

This content has been downloaded from IOPscience. Please scroll down to see the full text. 2015 Nucl. Fusion 55023001

(http://iopscience.iop.org/0029-5515/55/2/023001)

View the table of contents for this issue, or go to the journal homepage for more

Download details:

IP Address: 131.155.151.8

This content was downloaded on 12/01/2015 at $14: 24$

Please note that terms and conditions apply. 


\title{
Control of the tokamak safety factor profile with time-varying constraints using MPC
}

\author{
E. Maljaars ${ }^{1}$, F. Felici ${ }^{1}$, M.R. de Baar ${ }^{1,2}$, J. van Dongen ${ }^{2}$, \\ G.M.D. Hogeweij ${ }^{2}$, P.J.M. Geelen ${ }^{1}$ and M. Steinbuch ${ }^{1}$ \\ ${ }^{1}$ Eindhoven University of Technology, Mechanical Engineering, Control Systems \\ Technology, PO Box 513, 5600MB Eindhoven, The Netherlands \\ ${ }^{2}$ FOM Institute DIFFER, PO Box 1207, 3430BE Nieuwegein, The Netherlands \\ E-mail: e.maljaars@tue.nl
}

Received 13 March 2014, revised 16 September 2014

Accepted for publication 19 September 2014

Published 6 January 2015

\begin{abstract}
A controller is designed for the tokamak safety factor profile that takes real-time-varying operational and physics limits into account. This so-called model predictive controller (MPC) employs a prediction model in order to compute optimal control inputs that satisfy the given limits. The use of linearized models around a reference trajectory results in a quadratic programming problem that can easily be solved online. The performance of the controller is analysed in a set of ITER L-mode scenarios simulated with the non-linear plasma transport code RAPTOR. It is shown that the controller can reduce the tracking error due to an overestimation or underestimation of the modelled transport, while making a trade-off between residual error and amount of controller action. It is also shown that the controller can account for a sudden decrease in the available actuator power, while providing warnings ahead of time about expected violations of operational and physics limits. This controller can be extended and implemented in existing tokamaks in the near future.
\end{abstract}

Keywords: model predictive control, profile control, plasma scenarios, tokamaks, actuator sharing

(Some figures may appear in colour only in the online journal)

\section{Introduction}

Successful high performance tokamak operation simultaneously requires plasma stability at high values of the normalized pressure $\beta_{\mathrm{N}}$ as well as long energy confinement times $\tau_{E}$. Both confinement and stability are associated with the distribution of the current density in the plasma, equivalent to the safety factor profile $q(\rho)$. Achieving and maintaining a reference $q$-profile during a tokamak discharge in the presence of disturbances and uncertainties is one of the prospective main areas of research in currently operational tokamaks, ITER, and in other future long pulse devices such as JT60SA and Tore Supra WEST [1-4].

In present-day practice, actuator trajectories to achieve approximately the desired $q$-profile are chosen by the tokamak operator based on extensive experience. Those actuator trajectories are provided in open loop to the actuators (for instance the time traces of auxiliary heating and current drive and the desired plasma current). Recently, model-based optimization of the actuator trajectories has been proposed to achieve a target $q$-profile at the beginning of the flat-top phase [5-7]. In practice, the desired $q$-profile cannot be obtained or maintained without feedback control, due to the presence of model mismatches and disturbances.

Feedback controllers for the $q$-profile can either be designed using first principle models or using models obtained by system identification procedures. The models obtained by system identification (such as applied in [8]) are valid in the vicinity of a single operating point only. First principle models can be used in essence in an arbitrary range of operating points, including the ramp-up and ramp-down phase [9-11].

In the literature, a large number of first principle model based feedback control approaches to control the $q$-profile are proposed of which we mention here the most recent [12-22]. Most of these contributions take fixed actuator constraints passively into account, meaning that they are not included in the controller design, but a posteriori imposed by the use of e.g. an anti-windup loop. Handling of actuator constraints in the $q$-profile controller design itself is done in $[13,14,16]$, where actuator constraints are fixed.

Dynamic actuator sharing is required for the simultaneous control of magnetic and kinetic profiles/variables, neoclassical tearing modes (NTMs) etc using a common set of actuators [23]. A common example is electron cyclotron (EC) heating and current drive, that can be used for many of these tasks [24]. A supervisory controller may optimize the allocation of actuators to simultaneously satisfy a number of control and plant protection tasks. This implies that the available actuator power for profile control (and the distribution thereof) varies in time and hence the controller should take those real-timevarying limits actively into account. 


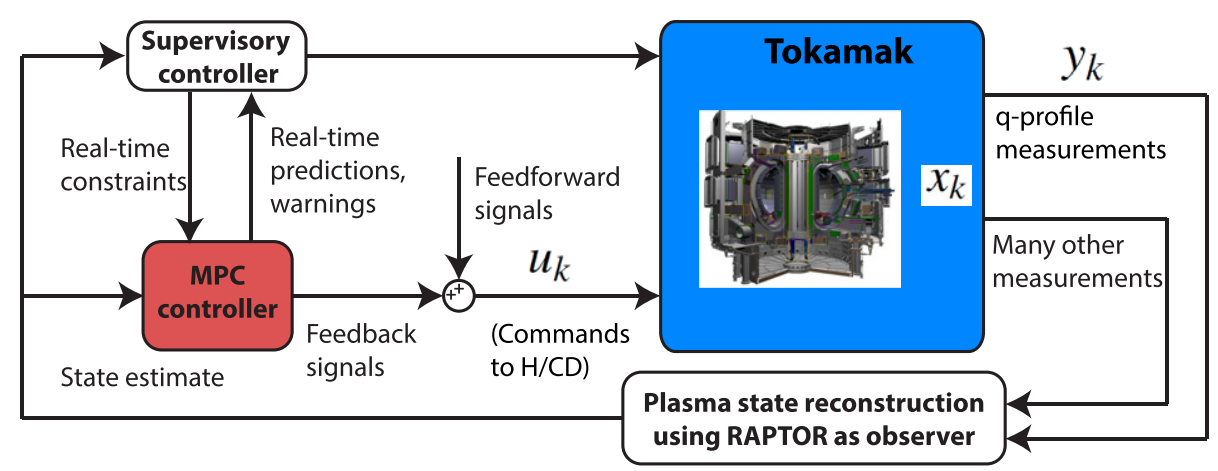

Figure 1. Envisioned implementation of the MPC controller in a tokamak. A supervisory controller receives real-time predictions and warnings of the MPC controller. It provides the MPC controller with real-time constraints. The MPC controller calculates the optimal feedback signal which is provided with the feedforward signal to the actuators in the tokamak. The plasma state is reconstructed from the available measurements using a state observer and fed to the MPC controller. A state observer based on RAPTOR is presented in [29].

Besides actuator limits, it would be desirable for a controller to ensure simultaneously that physics operational limits (e.g. limits on $\beta_{\mathrm{N}}$ and $l_{\mathrm{i}}^{(3)}$ ) and desired physics limits (e.g. $q(\rho)>1$ in the case of hybrid scenarios) are satisfied, as argued in e.g. [25]. Active handling of both actuator and plasma physics constraints simultaneously is not yet reported for the control of the $q$-profile in tokamak plasmas and is shown in this paper for the first time using a model predictive controller.

Model predictive control (MPC) is a general optimal control method which uses a predictive model to compute the control action and can deal routinely with actuator and state constraints [26-28]. MPC has already been proposed for $q$-profile control in $[13,14]$. The approach used in these references resulted in a non-linear optimization problem (which is computationally demanding). Moreover, the used models contained only the magnetic flux evolution equation and only static actuator constraints were considered.

The MPC controller in this work uses locally linearized models at each time step around a reference trajectory to minimize the tracking error in the presence of real-timevarying actuator and plasma physics constraints, model uncertainties and disturbances. The controller uses a quadratic optimization which is computationally less demanding and the locally linearized models contain the dynamic response of the poloidal magnetic flux and electron temperature.

The proposed feedback controller is designed to improve normal well-prepared operation of currently operational tokamaks by complementing feedforward actuator trajectories with feedback control around a predefined reference trajectory. The profile controller is intended to work under normal plasma behaviour with modest uncertainty and when very significant and unexpected changes occur in the plasma behaviour, e.g. high confinement loss or large impurity accumulation etc, than the priority is not in controlling the profiles but the supervisory system will switch to exception handling. Therefore the controller has not been tested for such off-normal situations.

Simulations using the non-linear plasma transport simulator RAPTOR [7] show the effectiveness of this approach. An L-mode plasma with ITER-like parameters and a low shear $q$-profile is simulated during the ramp-up and flattop phase with the desired plasma current $I_{\mathrm{p}}, \mathrm{NBI}$ and ECCD as actuators. We show effective control of the $q$-profile in the presence of model mismatches and time-varying actuator and plasma physics constraints. It is shown that real-time prediction of the $q$-profile evolution enables early warnings of plasma physics constraint violations. While the most important dynamics and underlying non-linearities from the 1D transport physics are taken into account, the method is fast enough to be implemented on the timescale of currently operational tokamaks.

This paper is organized as follows. In section 2 we present the methodology of the MPC controller design. The simulation setup is presented in section 3 . In section 4, we show performance and merits of this MPC controller for a number of typical cases. In section 5 we discuss the results and possible extensions of the current MPC design. The conclusion is provided in section 6 .

\section{Methodology}

In this section we describe the MPC controller design. First we introduce the context in which the controller will function and then the RAPTOR code, from which we obtain local linearized models used for the controller design, is briefly explained. The reader will subsequently be introduced to the concept of MPC and to the details of the control design up to the implementation in an algorithm.

\subsection{Controller context}

The envisioned implementation of the MPC controller in the larger context of a tokamak plasma control system is given in figure 1. A supervisory controller provides real-time constraints to the MPC controller. With these constraints the MPC controller calculates the optimal feedback action which is provided in combination with the feedforward signal to the actuators in the tokamak. From the available measurements the plasma state is reconstructed (either by a real-time observer [29] or by constrained equilibrium reconstruction) and fed to the MPC controller.

To systematically develop the MPC controller towards the envisioned implementation, the MPC controller is as a first step interfaced with the non-linear plasma transport simulator RAPTOR. This is illustrated in figure 2. This allows for a evaluation of the proposed control method in the non-linear plasma transport simulation of RAPTOR. The MPC controller is a state feedback controller [27], requiring access to the 


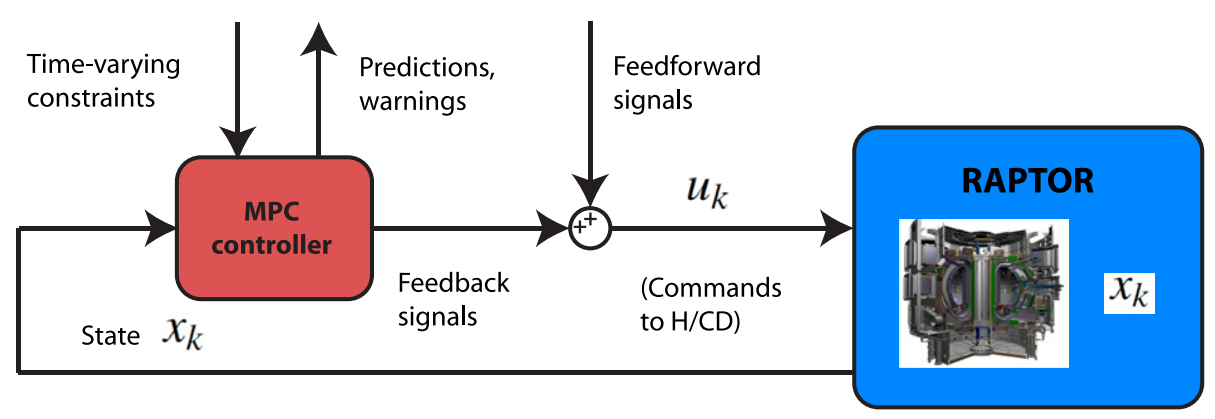

Figure 2. Simplified implementation as used in this work. The simulated plasma state is directly available to the MPC controller.

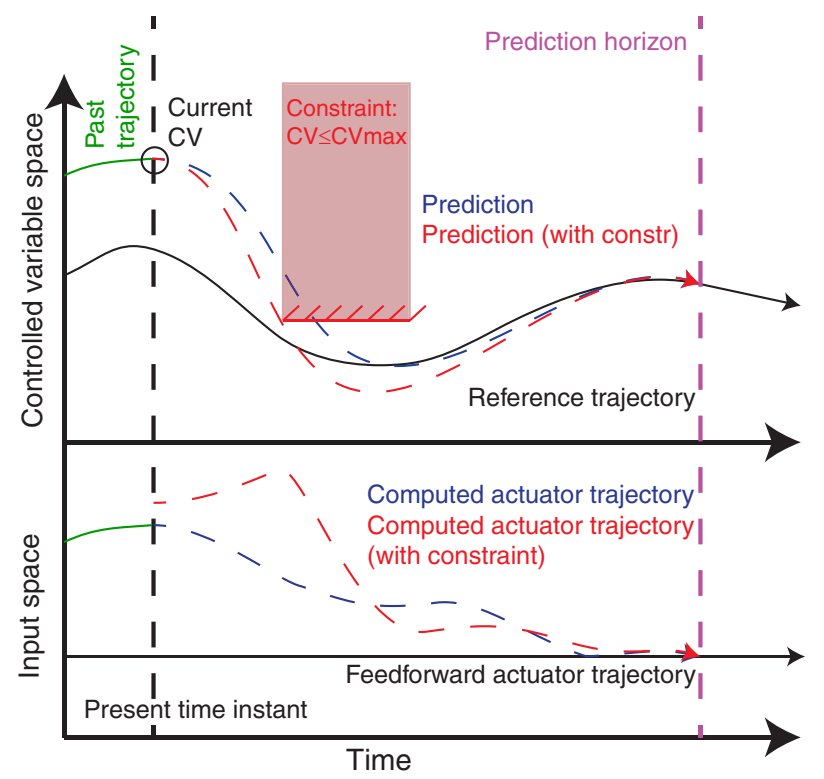

Figure 3. Illustration of MPC with single input and single controlled variable. Top: controlled variable trajectories; bottom: actuator trajectories. Feedforward actuator trajectory and its resulting nominal $\mathrm{CV}$ trajectory (that functions as reference) are given in black. Past trajectories of actuator input and controlled variable are given in green. MPC controller predicts until a given prediction horizon (magenta) what actuator trajectory (blue, bottom) is required in order to bring the controlled variable back to the reference trajectory (blue, top). In case of the presence of a controlled variable constraint, the MPC controller computes a future actuator trajectory (red, bottom panel) to handle this constraint (red, top panel).

actual state of the plasma, in this case, full knowledge of the plasma profiles. In this work we have direct access to the state of RAPTOR, which simplifies the implementation. The supervisory controller is not considered in this paper, instead time-varying constraints are manually provided to the MPC controller.

\subsection{RAPTOR: non-linear plasma transport simulator}

In this work we employ linearized models from RAPTOR inside the controller and use RAPTOR as simulator to test the controller. RAPTOR [7,11] is a control-oriented, physics-based 1D transport code that solves the simplified non-linear coupled transport of the electron temperature $T_{\mathrm{e}}$ and the poloidal magnetic flux $\psi$ as a function of the normalized square-root toroidal flux $\rho$, represented by partial differential equations (PDEs) [30]. Bootstrap current and neoclassical conductivity are calculated using the SauterAngioni equations [31,32]. Sources and sinks of thermal energy are modelled, including ohmic heating, and simple models for EC and neutral beam (NB) heating and current drive. Losses from bremsstrahlung, line radiation, and electron-ion heat exchange are included. The electron thermal diffusivity is computed using an empirical model, in this case the Bohm-gyroBohm transport model [33]. Impurities are not considered other than their effect via the $Z_{\text {eff }}$ profile, which is also prescribed. Another important assumption in RAPTOR is that the geometric terms in the transport equations, i.e. those terms that depend on the flux surface geometry, are chosen for one particular equilibrium and kept fixed thereafter. The total plasma current is imposed as boundary condition for the poloidal flux equation, while the electron temperature at the boundary is prescribed. In [34] is shown that by careful choices of what to exclude, RAPTOR is able to approach CRONOS [35] simulations of ITER in [36] within $\sim 15 \%$ or better, with $<3$ ms per time step.

Actuators considered in this work are the inductive and non-inductive heating and current drives (H\&CD). The controlled variables are the inverse $q$-profile $(\iota=1 / q)$ at several locations $\rho$ in the plasma. A state space description of the 1D plasma transport is used in which the internal state variables describe the electron temperature and the magnetic flux profiles at a particular moment in time. By discretization in both space and time, the following input, state and controlled variables $(\mathrm{CV})$ vectors can be introduced (as used in the simulations in this work):

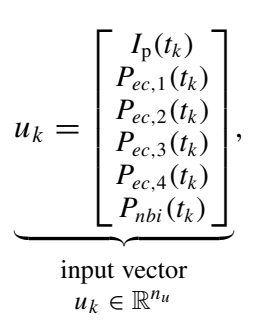$$
x_{k}=\underbrace{\left[\begin{array}{c}
x_{k} \in \mathbb{R}^{n_{x}} \\
\hat{y}_{\text {vector }}
\end{array}\right.}_{\left.\begin{array}{c}
\hat{T}_{\mathrm{e}_{\rho=0}}\left(t_{k}\right) \\
\vdots \\
\hat{T}_{\mathrm{e}_{\rho=1}}\left(t_{k}\right) \\
\hat{\psi}_{\rho=0}\left(t_{k}\right) \\
\vdots \\
\hat{\psi}_{\rho=1}\left(t_{k}\right)
\end{array}\right]}
$$

$$
\underbrace{z_{k}=\left[\begin{array}{c}
\iota_{\rho=0}\left(t_{k}\right) \\
\vdots \\
\iota_{\rho=1}\left(t_{k}\right)
\end{array}\right]}_{\text {CV vector }} .
$$

$\mathrm{CV}$ vector

$$
z_{k} \in \mathbb{R}^{n_{z}}
$$

The hats in the state vector denote that these are the finite element basis function coefficients instead of the actual values 
of $T_{\mathrm{e}}$ and $\psi$ [11]. In the simulations we used $n_{u}=6, n_{x}=64$, $n_{z}=16$, although $n_{x}=44$ would have been sufficient. Note that we calculate the internal state variables at a more fine $\rho$-grid than the controlled variables. This allows for more accurate solving of the transport while limiting the number of controlled variables. Using the discretization in both space and time the PDEs are formulated into a non-linear state update equation used to yield the next state $x_{k+1}$ from knowledge of the current state $x_{k}$ and actuator commands $u_{k}$ [7]:

$$
\tilde{f}_{k} \equiv \tilde{f}\left(x_{k+1}, x_{k}, u_{k}\right)=0 .
$$

This equation is solved in RAPTOR for each time instance $t_{k}$. The controlled variables are related to the state by the following controlled variable equation:

$$
z_{k}=h\left(x_{k}\right),
$$

which may provide many different controlled variables as a function of the state (e.g. inverse $q$-profile, $\beta_{\mathrm{N}}$, stored energy, magnetic shear). In this work we restrict ourselves to the inverse $q$-profile at different locations.

\subsection{Model predictive control}

The principle of MPC is illustrated and explained in figure 3. MPC solves at each moment in time an optimization problem to find the future actuator inputs up to a prediction horizon. This optimization problem involves a cost function (that represents e.g. the future tracking error) and constraints on actuators and states. MPC uses a process model of the dynamics to predict the future process behaviour. Only the computed actuator commands for the next time instant are implemented and the controller solves on the next step a new optimization problem with the updated state.

An MPC controller requires a model that relates the states and controlled variables at the next time instant to the current state and future actuator commands. The type of model required depends on which formulation of MPC that will be used. Non-linear MPC is available [37], but has a number of drawbacks with respect to linear MPC. Linear MPC is more established, and the use of a linear model in combination with a quadratic cost function and linear constraints results in a quadratic programming (QP) problem, while non-linear MPC requires the optimization of a non-linear optimization problem which is computationally more demanding. Moreover, the solution of a QP problem is a global constrained minimum whereas non-linear optimization problems may have local minima.

The controller should run about five times faster than the fastest process time scale that dominates the influence of inputs on the controlled variable evolution or the evolution of constrained quantities. For profile control that includes also kinetic quantities, this is the energy confinement time, which in medium-sized tokamaks such as ASDEX-Upgrade is $\sim 50 \mathrm{~ms}$. This implies that for implementation on a device such as ASDEX-Upgrade, the computational time of the MPC controller is limited to approximately $10 \mathrm{~ms}$. It is therefore necessary to reduce the online computational cost as much as possible, and this motivates the use of linear MPC techniques and hence the formulation of linear models.
We benefit from the fact that tokamak discharges follow a predefined sequence of current ramp-up, flat-top and current ramp-down with the corresponding predefined auxiliary actuator trajectories. These actuator trajectories will nominally (i.e. in the absence of disturbances and model mismatches) result in a particular feedforward state evolution. We therefore assume that profiles in RAPTOR also evolve nominally along pre-calculated trajectories $\left(u_{k}^{o}, x_{k}^{o}, z_{k}^{o}, \forall k\right)$. An actuator trajectory with its corresponding state and controlled variable trajectory can thus be specified as a nominal trajectory, where the nominal controlled variable trajectory may function as time-varying reference. The non-linear dynamics can then be linearized at each time instant $t_{k}$ resulting in a sequence of linear time-varying (LTV) models that can be used in a linear MPC controller to track this reference trajectory.

Hereafter we will derive the linearizations, define the prediction model, the cost function and constraints and apply some strategies to reduce the computational cost.

\subsection{Linearizations around trajectory}

Linearizations around the nominal trajectory are obtained offline by defining an infinitesimally small perturbation in the state $\tilde{x}_{k}=x_{k}-x_{k}^{o}$ and input $\tilde{u}_{k}=u_{k}-u_{k}^{o}$. The dynamics of $\tilde{x}_{k}$ can then be derived using the Taylor expansion of (1):

$0=\tilde{f}\left(x_{k+1}^{o}, x_{k}^{o}, u_{k}^{o}\right)=\frac{\partial \tilde{f}}{\partial x_{k+1}} \tilde{x}_{k+1}+\frac{\partial \tilde{f}}{\partial x_{k}} \tilde{x}_{k}+\frac{\partial \tilde{f}}{\partial u_{k}} \tilde{u}_{k}$.

A so-called LTV state space model [38] can now be derived by solving (3) for $\tilde{x}_{k+1}$ and linearizing the controlled variables equation (2):

$$
\begin{gathered}
\tilde{x}_{k+1}=A_{k} \tilde{x}_{k}+B_{k} \tilde{u}_{k}, \\
\tilde{z}_{k}=C_{k} \tilde{x}_{k}+D_{k} \tilde{u}_{k},
\end{gathered}
$$

with the state space matrices defined as:

$$
\begin{gathered}
A_{k}=\left(\frac{\partial \tilde{f}}{\partial x_{k+1}}\right)^{-1} \frac{\partial \tilde{f}}{\partial x_{k}}, \quad B_{k}=\left(\frac{\partial \tilde{f}}{\partial x_{k+1}}\right)^{-1} \frac{\partial \tilde{f}}{\partial u_{k}}, \\
C_{k}=\frac{\partial h}{\partial x_{k}}, \quad D_{k}=\frac{\partial h}{\partial u_{k}}=0 .
\end{gathered}
$$

RAPTOR provides the underlying Jacobians at each time step around the nominal trajectory. Practically, the Jacobian $\left(\partial \tilde{f} / \partial x_{k+1}\right)$ is invertible for all physically relevant values of the state $x_{k}$.

Figure 4 illustrates the linearizations around a predefined trajectory and shows how the linearized models allow for an accurate description of the dynamics around the nominal trajectory within a validity region. The applicability of this approach will be verified in section 3.4.

\subsection{Prediction model}

The MPC controller uses a prediction model to relate the future states and controlled variables to the current state and future actuator commands. We can define a prediction model to 


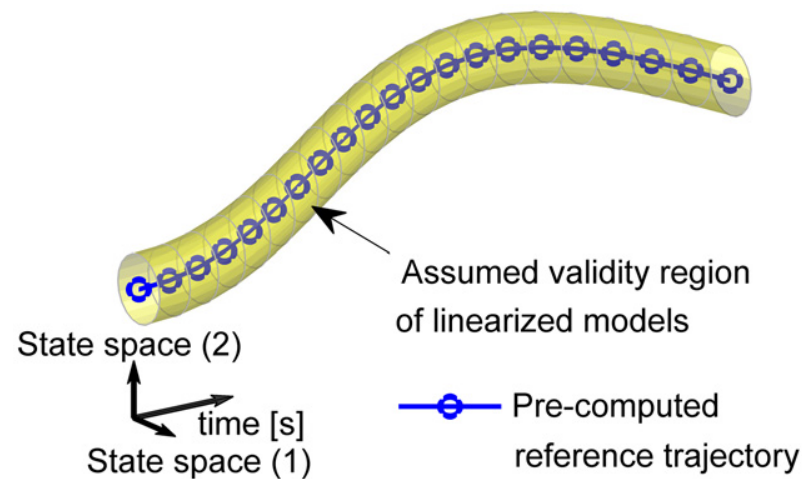

Figure 4. Illustration of using linearizations around pre-calculated trajectory in state space. At each moment in time (denoted by dots) a linearized state space model is derived. The yellow contour around the pre-calculated trajectory illustrates that each linearized state space model has a limited validity region (each indicated by circle). Together, linearized models with their validity region allow for an accurate description of the dynamics around a nominal trajectory.

predict $N$ steps ahead, where $N$ is called the prediction horizon. First we introduce the following stacked vectors:
$\tilde{U}_{k, N}=\left[\begin{array}{c}\tilde{u}_{k} \\ \tilde{u}_{k+1} \\ \vdots \\ \tilde{u}_{k+N}\end{array}\right]$,

Future inputs

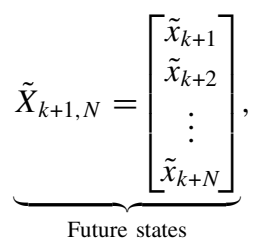

Future states

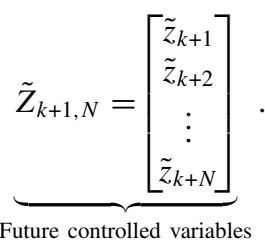

Future controlled variables
Using the same notation we can express

$$
\begin{aligned}
& U_{k, N}=U_{k, N}^{0}+\tilde{U}_{k, N}, \\
& X_{k+1, N}=X_{k+1, N}^{0}+\tilde{X}_{k+1, N}, \\
& Z_{k+1, N}=Z_{k+1, N}^{0}+\tilde{Z}_{k+1, N} .
\end{aligned}
$$

By using the obtained linearized state space models at each moment in time, we can write the future state deviations $\tilde{X}_{k+1, N}$ and future controlled variable deviations $\tilde{Z}_{k+1, N}$ as

$$
\begin{aligned}
\tilde{X}_{k+1, N} & =\Gamma_{A_{k, N}} \tilde{x}_{k}+\Gamma_{B_{k, N}} \tilde{U}_{k, N}, \\
\tilde{Z}_{k+1, N} & =\Gamma_{C_{k, N}} \tilde{x}_{k}+\Gamma_{D_{k, N}} \tilde{U}_{k, N} .
\end{aligned}
$$

The matrices $\Gamma_{A_{k, N}}, \Gamma_{B_{k, N}}, \Gamma_{C_{k, N}}$ and $\Gamma_{D_{k, N}}$ can be constructed from $N$ different linearized models from RAPTOR and are given in section A.1 in the appendix. The matrices are computed offline to reduce the online computational cost. The reader is referred to [39] for a full derivation of this prediction model. In practice, the time interval between predictions of future states and controlled variables is increased by deleting some of the rows of the prediction matrices (see section 2.8).

\subsection{Actuator and state constraints}

Constraints can be imposed on the individual actuators (for example the desired plasma current $I_{\mathrm{p}}^{\text {des }}$ is both constrained in amplitude and in ramp-rate) and on combinations of actuators (for example the available EC power to the different beams). As shown in section A.2.1 in the appendix, all the actuator constraints can be formulated as time-varying linear inequality constraints on the future input deviations $\tilde{U}_{k, N}$ :

$$
A_{\text {inp }} \tilde{U}_{k, N} \leqslant b_{\text {inp }, k} .
$$

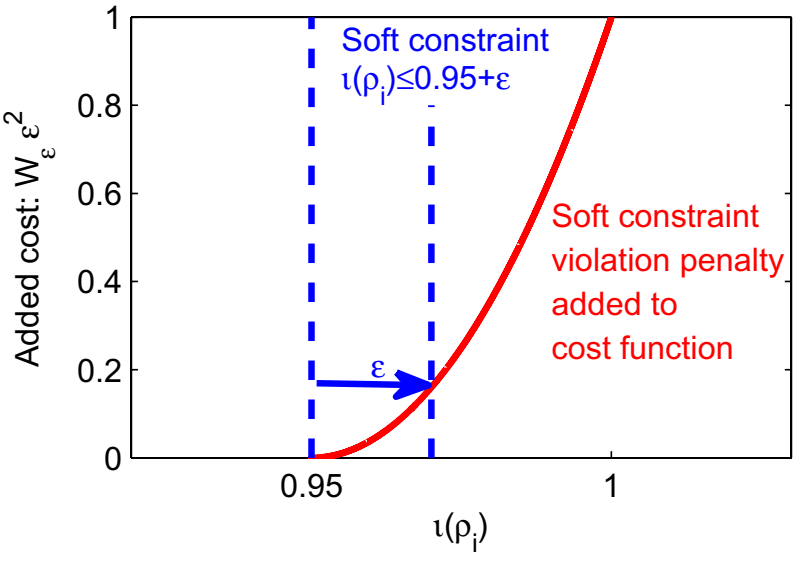

Figure 5. Illustration of using a soft state constraint $\iota(\rho) \leqslant 0.95+\varepsilon$ instead of a hard state constraint $\iota(\rho)<1$. Exceeding $\iota(\rho)=0.95$ is allowed, but the term $W_{\varepsilon} \varepsilon^{2}$ penalizes entering this soft constraint region.

The state constraints limit a certain function of the states. An example of a state constraint is that if we want to avoid sawteeth, we ask to ensure $q(\rho)>1(\iota(\rho)<1)$ at all times. Since we have $D=0$ in our model, constraints on controlled variables can be rewritten as linear inequality constraints on the states.

Hard state constraints are discrete (i.e. they are satisfied or violated). It is important to note that operating in the vicinity of a hard state constraint can be dangerous. Being in the vicinity of the hard constraint, a disturbance may result in violating the constraint and this also implies that the controller may find no actuator trajectory to stay within the constraint.

A common approach in MPC is to use soft state constraints in which state constraint violation is allowed, but the violation is penalized in the controller cost function. The parameter $\varepsilon$ indicates the soft state constraint violation and $\varepsilon>0$ when the soft state constraint is violated. The penalty in the cost function is added as $W_{\varepsilon} \varepsilon^{2}$. The scalar $W_{\varepsilon}$ sets the softness or stiffness of the soft state constraint.

In figure 5 it is illustrated how a soft state constraint $\iota(\rho) \leqslant 0.95+\varepsilon$ can be applied to avoid operation in the vicinity of the hard constraint $\iota(\rho) \leqslant 1$. The soft constraint violation $\varepsilon$ can be monitored in real time and reported to a supervisory controller that may anticipate on this information. In section A.2.2 it is shown that all soft state constraints can be cast as time-varying linear inequality constraints on the future input deviations $\tilde{U}_{k, N}$ and the soft constraint violation $\varepsilon$ :

$$
A_{\text {state }}\left[\begin{array}{c}
\tilde{U}_{k, N} \\
\varepsilon
\end{array}\right] \leqslant b_{\text {state }, k}
$$

\subsection{Cost function}

The controller has the objective to minimize the future error while avoiding too aggressive control actions and entering the soft constraint region. This can be expressed in the cost function $J_{k}$ as follows:

$$
J_{k}=\tilde{Z}_{k+1, N}^{T} Q \tilde{Z}_{k+1, N}+\Delta \tilde{U}_{k, N}^{T} R_{\Delta \tilde{U}} \Delta \tilde{U}_{k, N}+W_{\varepsilon} \varepsilon^{2},
$$



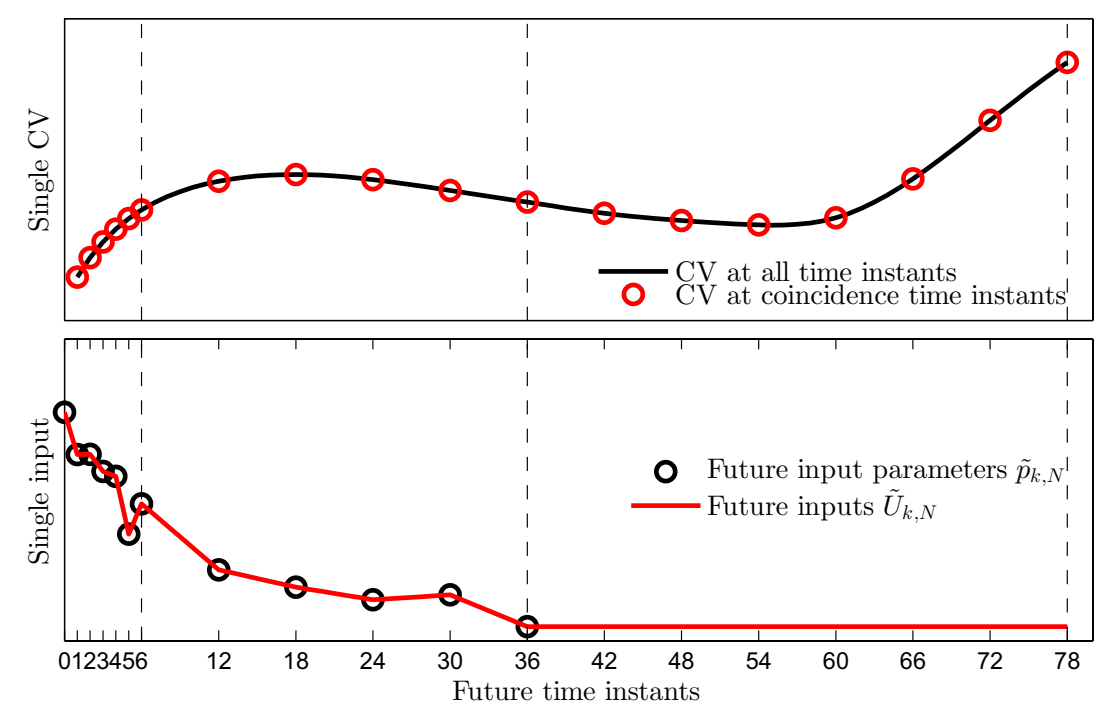

Figure 6. Illustration of reducing the computational cost using concepts of input parametrization and coincidence points as used in the simulation results. Figure illustrates for one actuator and one controlled variable. Input parametrization (bottom panel): a small number of parameters $\tilde{p}_{k, N}(\mathrm{o})$ are used to define a complete future input sequence $\tilde{U}_{k, N}(-)$. Coincidence points (top panel): the states and controlled variables are only predicted at a subset of time instants in the prediction horizon and desired to coincide with a reference trajectory only at these coincidence time instants. The CV at all time instants (-) is shown together with the CV at the coincidence time instants (o). The settings are explained in section 3.5 .

where

$$
\Delta \tilde{U}_{k, N}=\left[\begin{array}{c}
\tilde{u}_{k+1}-\tilde{u}_{k} \\
\vdots \\
\tilde{u}_{k+N}-\tilde{u}_{k+N-1}
\end{array}\right]=\Gamma_{\Delta} \tilde{U}_{k, N}
$$

and $\Gamma_{\Delta}$ being a difference matrix operator. In the cost function (10) we identify the following weights:

- $Q$ is a diagonal performance weight on the future error norm;

- $R_{\Delta U}$ is a diagonal input difference weight to avoid aggressive control actions;

- $W_{\varepsilon}$ is a weight that defines the flexibility of the soft constraint.

Note that by choosing this structure of the cost function, the actuator trajectories remain unchanged in the absence of model mismatches, disturbances and more strict constraints. This is desirable as more complete knowledge is available offline in the optimization of the feedforward actuator trajectories than online in the MPC controller.

Tuning of the weights in an MPC controller in the absence of model mismatches and active constraints is straightforward and intuitive. However, it is known from literature (e.g. [26]) that the presence of (many) active constraints and model mismatches makes the effect of tuning parameters less clear. Therefore performing a set of representative simulations is required prior to experiments in order to obtain the optimal settings of the controller as a compromise between performance and robustness under the circumstances (e.g. model mismatches and active constraints) that can be expected in the actual experiments. The chosen control settings are discussed in section 3.5.

\subsection{Strategies to reduce the online computational cost}

In order to meet the computational requirements, the optimization problem can be made more compact (less optimization variables and linear constraints) using concepts from literature (e.g. [26, 28, 40]). We will discuss here the use of input parametrization and the reduction of the number of predicted states, controlled variables and constraints via the concept of coincidence points.

We parametrize each future actuator input sequence in $\tilde{U}_{k, N}$ by a relative small number of unknown parameters. In fact, the parameters $\tilde{p}_{k, N}$ are the inputs at some specified time instants (nodes), where we linearly interpolate between those nodes for the remaining time instants. In addition, we keep all inputs constant after a so-called control horizon $N_{c}$, which is common in MPC practice.

The linear mapping between a future input parameter sequence $\tilde{p}_{k, N}$ and the future input sequence $\tilde{U}_{k, N}$ can be written as

$$
\tilde{U}_{k, N}=P_{\text {map }} \tilde{p}_{k, N},
$$

where the parameterization mapping matrix $P_{\text {map }} \in$ $\mathbb{R}^{(N+1) \cdot n_{u} \times n_{p}}$ is fixed and chosen offline.

To reduce the number of predicted states, controlled variables and constraints, we use the concept of coincidence points. Herein, the reference and predicted controlled variables are desired to coincide only at a limited number of time instants in the prediction horizon. We choose to compute the predicted states and controlled variables only at this subset of time instants and additionally state constraints are only imposed at these time instants. By choosing the time between coincidence points not larger than the time between the nodes in the input parametrization, using linear interpolation and considering the diffusive nature of the plasma profile dynamics, we can safely assume that no significant excursions of the states and controlled variables will occur in between those coincidence points.

The concept of input parametrization and coincidence points is illustrated in figure 6 for one actuator and one controlled variable, using the settings as will be discussed in section 3.5. 
Algorithm 1. Offline algorithm to prepare for online QP problems.

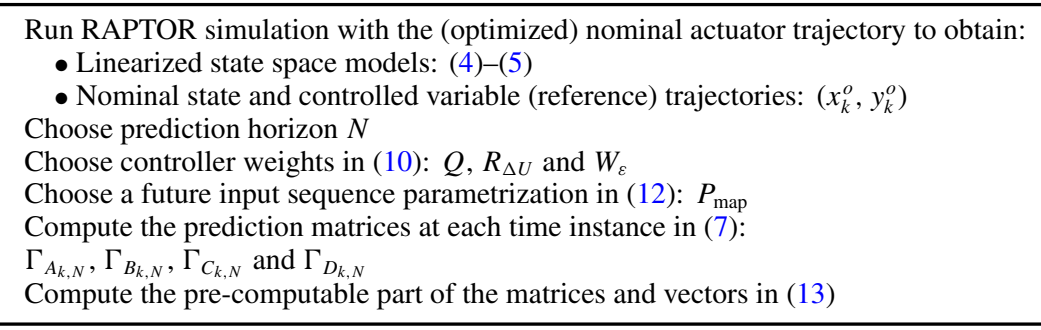

Algorithm 2. Online algorithm inside MPC controller

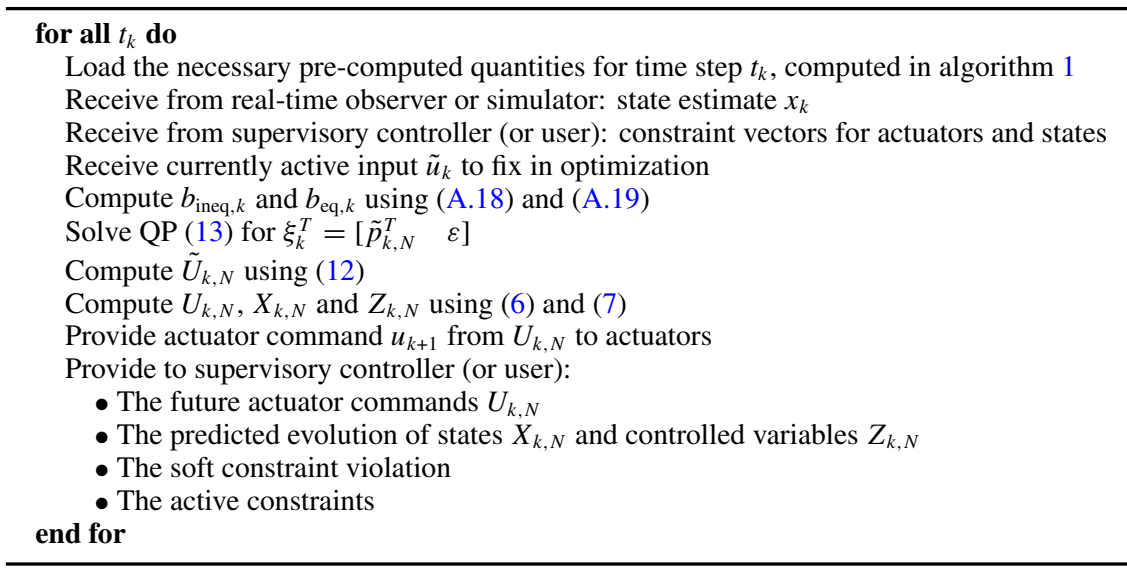

\subsection{Quadratic programming}

The future input deviations $\tilde{U}_{k, N}$ can be found by minimizing the cost function (10) subjected to the constraints (8) and (9). Note that the first input $\tilde{u}_{k}$ is the currently active input on the system and hence fixed in the optimization via equality constraints. Together, this can be rewritten using the prediction model (7) and CVP (12) as an online QP problem at each time instant $t_{k}$ :

$$
\begin{array}{cl}
\underset{\xi_{k}}{\operatorname{minimize}} & \frac{1}{2} \xi_{k}^{T} H_{k} \xi_{k}+\tilde{x}_{k}^{T} F_{k} \xi_{k}, \\
\text { subject to } & A_{\text {ineq }, k} \xi_{k} \leqslant b_{\text {ineq }, k}, \\
& A_{\text {eq }} \xi_{k}=b_{\text {eq }, k},
\end{array}
$$

where $\xi_{k}^{T}=\left[\begin{array}{ll}\tilde{p}_{k, N}^{T} & \varepsilon\end{array}\right]$. The matrices $H_{k}, F_{k}, A_{\text {ineq, } \mathrm{k}}$ and $A_{\text {eq }}$ are computed offline and the vectors $b_{\text {ineq, } k}$ and $b_{\text {eq }, k}$ are externally provided by the user or set in real time by a supervisory controller. All are defined in section A.4 in the appendix.

QP is computationally cheap and has a unique constrained solution. Many algorithms exist to solve the QP problem (13) online, that can handle large number of free parameters and abundant linear constraints, e.g. [41, 42]. Using QPC [41], results in a computational time per time instant of less than $8 \mathrm{~ms}$ in the simulation cases of section 4 on a standard offthe-shelf laptop. This is fast enough for implementation on an existing tokamak, even more when one considers using dedicated hardware.

\subsection{Summary of implementation}

The implementation of the MPC controller consists of an offline part and an online (real-time) part. To reduce the online computational burden, as many steps as possible are done offline. Offline, the steps are performed as given in algorithm 1 .

Once the offline steps have been done, the online part can start during the simulation or experiment. Inside the MPC controller, at each time step, algorithm 2 is used.

\section{Simulation setup}

In this section we describe the simulation setup we developed to test the MPC controller. A plasma scenario is set up in RAPTOR with ITER parameters [34]. It is not the objective of these simulations to provide quantitative estimates of the ITER performance or controllability of a particular scenario, but to illustrate the potential of an MPC controller for profile control. First we introduce the physics model in RAPTOR, then an optimized nominal trajectory will be explained, after which we will investigate the performance of the linearized models as used in the controller and end with the controller settings as used in the simulations section.

\subsection{Physics model and settings in RAPTOR}

A CRONOS simulation of an ITER L-mode ramp-up in [36] is used to supply the initial profiles of $T_{\mathrm{e}}$ and $\psi$, the prescribed profile evolutions of $n_{\mathrm{e}}, n_{\mathrm{i}}$ and $Z_{\text {eff }}$ as well as those quantities in the transport equations depending on the $2 \mathrm{D}$ magnetic equilibrium. Although the controller design is in principle applicable to any operating mode, we restrict ourselves in this work to L-mode simulations. The $\mathrm{L}-\mathrm{H}$ transition is not modelled in these simulations. It is assumed that we remain always in L-mode, although we slightly exceed the predicted $\mathrm{L}-\mathrm{H}$ transition power threshold for this density evolution as reported in [36]. 
In the present work, the $2 \mathrm{D}$ magnetic equilibrium is assumed to be fixed in time. The simulation starts at $20 \mathrm{~s}$, when the plasma current equals 4.7 MA. The ion temperature profile evolution is a fixed scaling of the electron temperature evolution, i.e.: $T_{\mathrm{i}}(\rho, t)=f_{T_{\mathrm{i}}}(\rho) T_{\mathrm{e}}(\rho, t)$. As most heating in the core is provided to the electrons, rather than the ions, $f_{T_{\mathrm{i}}}(\rho)$ is chosen as a linear function, increasing from 0.6 at the centre until 1 at the edge. As RAPTOR is using an implicit time-discretization scheme, large time steps are allowed while remaining numerically stable at the expense of some loss of accuracy for larger time steps. For this plasma all dominant time scales in the input-controlled variable behaviour are larger than $1 \mathrm{~s}$. Therefore we chose in the first second a time step of $50 \mathrm{~ms}$ to simulate the initial phase, for the remainder a time step of $1 \mathrm{~s}$ was chosen. Open-loop simulations with smaller time steps produced the same results up to a small error, confirming that the chosen time steps are small enough.

The controlled actuators considered are the desired plasma current $I_{\mathrm{p}}^{\mathrm{des}}$ and the power to four EC beams deposited at different locations as given below:

\begin{tabular}{cccc}
\hline Beam & Location $\rho$ & Type EC & Gaussian full width in $\rho[]$ \\
\hline 1 & 0 & co & 0.2 \\
2 & 0 & counter & 0.2 \\
3 & 0.2 & co & 0.1 \\
4 & 0.4 & co & 0.1 \\
\hline
\end{tabular}

The combination of equatorial launcher and the upper port launchers as given in the ITER design allows for EC-deposition in the region between $\rho=0$ and $\rho \approx 0.6$, where we use here deposition up to $\rho=0.4$. The EC-beam power and current density deposition is modelled by Gaussian profiles where the current drive efficiency scales with $T_{\mathrm{e}} / n_{\mathrm{e}}$. The nominal flat-top value is $\sum_{i=1}^{4} P_{\mathrm{EC}, i}=20 \mathrm{MW}$. It is explicitly assumed that the power will be distributed over the equatorial launcher and the upper port launchers by a dedicated low level controller that is outside the scope of this contribution. The off-axis NBI heating and current drive is modelled using a pencil beam model [34] and the NBI power is fully prescribed, ramping between 60 and $70 \mathrm{~s}$ from zero to its flat-top value of $16.5 \mathrm{MW}$, i.e. half of the available NBI power.

\subsection{Constraints}

We define here a set of actuator and plasma physics constraints that is imposed in the design of the nominal trajectory and in the MPC controller. The actuator constraints are:

- $0 \leqslant I_{\mathrm{p}} \leqslant 10 \mathrm{MA}$, during flat-top $6 \leqslant I_{\mathrm{p}} \leqslant 8 \mathrm{MA}$;

- $-0.1 \mathrm{MA} \mathrm{s}^{-1} \leqslant \frac{\mathrm{d} I_{\mathrm{p}}}{\mathrm{d} t} \leqslant 0.1 \mathrm{MA} \mathrm{s}^{-1}$;

- $P_{\mathrm{EC}, i} \geqslant 0 \mathrm{MW}, \forall i \in[1,2,3,4]$;

- $\Sigma P_{\mathrm{EC}} \leqslant 25 \mathrm{MW}$.

A soft state constraint is added as $\iota(\rho) \leqslant 0.95+\varepsilon$. In the design of the nominal trajectory the hard constraint $\iota(\rho)<$ 0.95 is added together with the requirement $I_{\mathrm{p}}\left(t_{\mathrm{end}}\right) \geqslant 7 \mathrm{MA}$.

Large and steep variations of the plasma current $I_{\mathrm{p}}$ during the flat-top phase are seen to induce MHD-activity and problems with the vertical stability control system, both related to exceeding limits on $l_{\mathrm{i}}^{(3)}$. Therefore the plasma current $I_{\mathrm{p}}$ is in present-day operation not often varied during the flat-top phase. However, imposing strict requirements on $I_{\mathrm{p}}$ during the flat-top phase maintains the plasma within the limits on $l_{\mathrm{i}}^{(3)}(t)$ and allows for using $I_{\mathrm{p}}$ as a feedback actuator. Alternatively, (linearized) soft state constraints may be imposed directly on $l_{\mathrm{i}}^{(3)}(t)$.

\subsection{Optimized nominal trajectory}

In principle, the nominal feedforward actuator trajectories can be determined by tokamak operators. Here, the feedforward actuator trajectories and the corresponding state and controlled variables trajectories are calculated following the numerical optimization described in [7].

The nominal trajectory optimization aims at achieving any $\iota$-profile which is stationary at the beginning of the flat-top phase, while staying within the actuator and plasma physics constraints as defined in section 3.2. The flat-top phase starts after $120 \mathrm{~s}$. The loop voltage profile $U_{\mathrm{pl}}(\rho)$ must be as flat as possible after $120 \mathrm{~s}$, meaning that the ohmic current redistribution has almost stopped and therefore the $\iota$-profile is stationary. The optimization cost function contains therefore solely a penalty on the gradient of the final loop voltage profile $U_{\mathrm{pl}}(\rho)$.

In our simulations, it was not possible to obtain a stationary $q$-profile at the beginning of the flat-top within the constraints with a flat-top plasma current $I_{\mathrm{p}}$ of more than $7 \mathrm{MA}$. This is mainly caused by the lack of the bootstrap current and low current drive efficiency due to low temperature in L-mode. We noticed that the obtainable flat-top plasma current $I_{\mathrm{p}}$ is also sensitive to the settings of the NBI actuator model, that was not yet benchmarked against other NBI models.

The optimized nominal trajectories for the current and EC-power distribution are shown in figures 7(a) and 7(e)-(h) respectively. The prescribed NBI power and the total EC power are shown in figure $7(b)$. The plasma current $I_{\mathrm{p}}$ is ramped up quickly until $40 \mathrm{~s}$, after which the numerical optimization calculates a small current overshoot at $100 \mathrm{~s}$. Such overshoots have been reported to be beneficial for achieving a stationary flat $\iota$-profile [7, 36, 43].

The optimization uses the flexibility in the EC-power allocation. At most times, the counter-ECCD power (figure $7(f)$ ) significantly exceeds the co-ECCD power (figure 7(e)). This implies that during such phases, a resultant negative central current is driven by these two EC beams. This helps to flatten the $U_{\mathrm{pl}}$-profile and prevents violating the constraint $\iota \leqslant 0.95$.

The EC beam at $\rho=0.2$ (figure $7(g)$ ) provides significant heating and current drive during the ramp-up phase. Its peak power occurs just before the NBI power (figure $7(b)$ ) is ramped up between 60 and $70 \mathrm{~s}$. The EC-power deposition at $\rho=0.4$ (figure $7(h)$ ) is ramped up until finally all available EC power is provided at this location $(20 \mathrm{MW})$ at $120 \mathrm{~s}$. The EC power is reduced during and just after the ramp-up of the NBI power as shown in figure $7(b)$. The prescribed ramp-up of central electron and ion densities is given in figure $7(d)$. The resulting central ion and electron temperatures $T_{\mathrm{i}, 0} T_{\mathrm{e}, 0}$ are shown in figure $7(c)$. Note that central temperatures reach their maxima after $40 \mathrm{~s}$.

At the times indicated by the grey dotted lines in the top panels $(60,70,100$ and $120 \mathrm{~s})$, the resulting profiles of $\iota, T_{\mathrm{e}}$, 

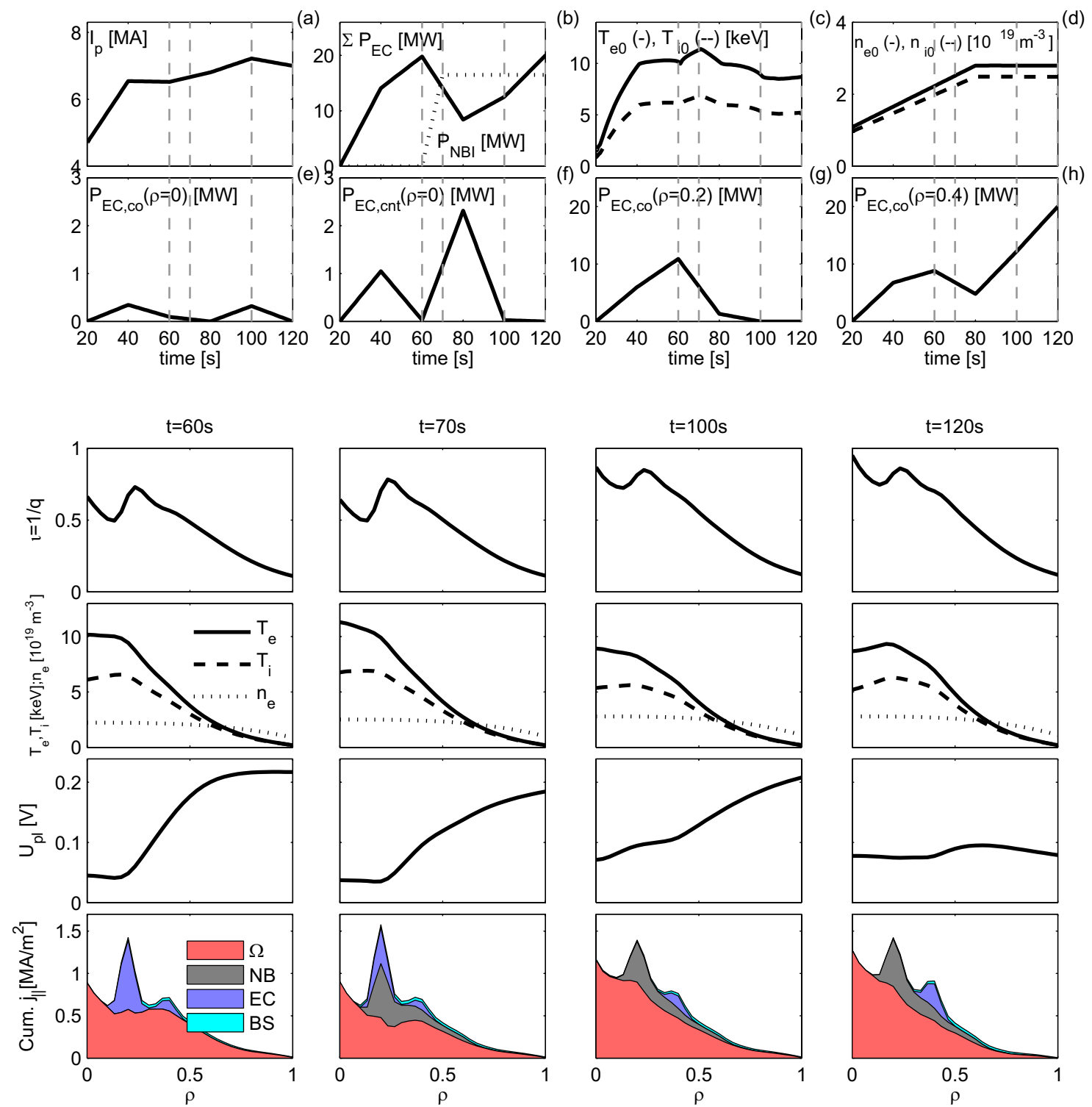

Figure 7. Optimized nominal trajectories for achieving stationary $\iota$-profile at the beginning of the flat-top phase in L-mode, while satisfying actuator and plasma physics constraints. Top panels show the time evolution of optimal actuator commands of the plasma current $I_{\mathrm{p}}(a)$, four EC beams $(e)-(h)$ and corresponding total EC power $(b)$, central temperatures $(c)$ and central densities $(d)$. The prescribed evolution of NBI power is also shown in $(b)$. Bottom panels show the profiles $\iota, T_{\mathrm{e}, \mathrm{i}}, n_{\mathrm{e}}, U_{\mathrm{pl}}$ and components of $j_{\|}$at $60,70,100$ and $120 \mathrm{~s}$. Note $I_{\mathrm{p}}$ $(120 \mathrm{~s})=7 \mathrm{MA}$, that is the maximum achievable plasma current in our simulations for achieving a stationary $\iota$-profile within the given constraints in L-mode.

$T_{\mathrm{i}}, n_{\mathrm{e}}, U_{\mathrm{pl}}$ and the components of the parallel current density $j_{\|}$are shown in the bottom panels of figure 7 . The $\iota$-profile increases smoothly to the final profile at $120 \mathrm{~s}$, which is close to the constraint $\iota(\rho)<0.95$. The temperature profile peaks at the centre after the ramping-up of the NBI beam $(70 \mathrm{~s})$ and the increasing power to the counter EC beam at $\rho=0$. The temperature profile afterwards (100 and $120 \mathrm{~s})$ widens and drops in the centre after the increasing concentration of all EC power at $\rho=0.4$. The $U_{\mathrm{pl}}$-profile evolves towards the nearly flat profile at $120 \mathrm{~s}$. Finally, the evolution of the cumulative current density profile is shown. The inward diffusion of the ohmic current density can be noticed which follows after $120 \mathrm{~s}$ the typical conductivity profile (corresponding to the flat $U_{\mathrm{pl}}$-profile). The NBI current density appears after $70 \mathrm{~s}$. Also the EC current density active at different locations can be noticed clearly. The bootstrap current is hardly visible and is only $6 \%$ of the plasma current in flat-top.

\subsection{Validity of linearized models}

The MPC controller approximates the non-linear plasma behaviour as a linearized system at each moment in time. Therefore, we now analyse the validity of the linearized models around the designed nominal trajectory by open-loop simulations. Special emphasis is put on the time evolution of the differences between the simulated outputs of the linearized and non-linear models as this is indicative for the expected prediction error in the MPC controller. A comparison with a single linear model is also included.

For this purpose the responses of the linearized and nonlinear models to a perturbation input vector are compared in 

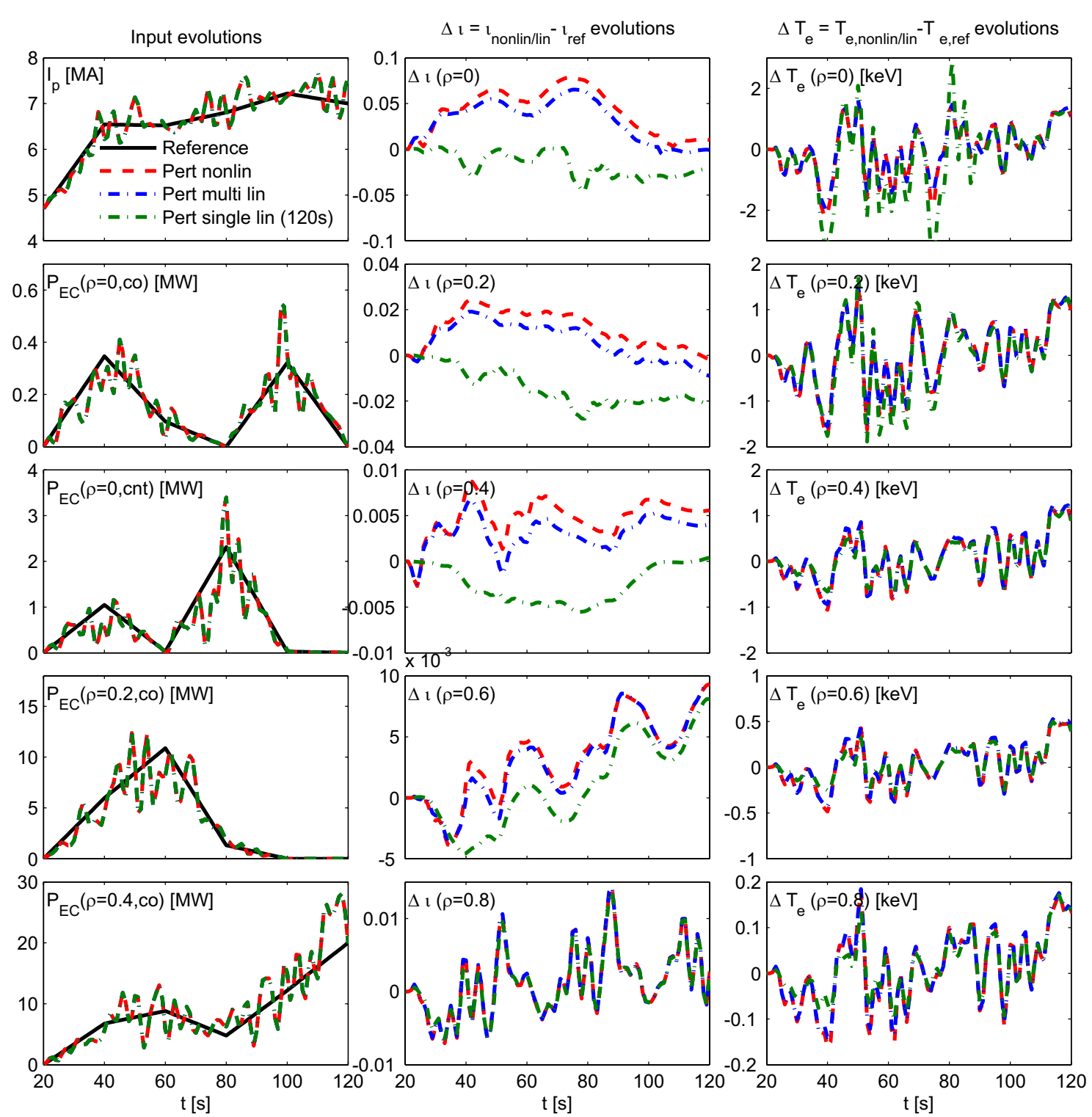

Figure 8. Comparison responses of linearized and non-linear models. Column 1: actuator evolutions of plasma current $I_{\mathrm{p}}$ and four EC powers for nominal case (-) and sum of sinusoids inputs on top of nominal trajectory for non-linear (- $)$ and cases using linearizations at each moment in time (--) and a single linearization (---). Column 2: response to perturbation inputs of $\Delta \iota$-profile. Column 3: effect of added perturbation inputs on $\Delta T_{\mathrm{e}}$-profile. Note the far more accurate description by multiple linearized models of both the $T_{\mathrm{e}}$-profile and $\iota$-profile with respect to a single linear model.

the following cases:

- Non-linear model (RAPTOR): the perturbation input vector $\tilde{u}_{k}$ is added to the nominal input vector $u_{k}^{\text {ref }}$ and fed to RAPTOR. Comparison of the outputs of RAPTOR for the case with perturbation and without perturbation yields $\Delta T_{\mathrm{e}, \text { nonlin }}$ and $\Delta \iota_{\text {nonlin }}$.

- Multiple linearized models: the perturbation input vector $\tilde{u}_{k}$ is fed into the models locally linearized at each moment in time which yields $\Delta T_{\mathrm{e} \text {,multi lin }}$ and $\Delta \iota_{\text {multi lin }}$.

- Single linear models: the perturbation input vector $\tilde{u}_{k}$ is fed into a single linear model (taken at end of ramp-up) which yields $\Delta T_{\mathrm{e}, \text { single lin }}$ and $\Delta \iota_{\text {single lin }}$.

The results are given in figure 8 . Random sum of sinusoids with a limited frequency band (often used in system identification [44]) are used for the verification and are shown in column $1(--),(-\cdot-)$ and (---) on top of the nominal actuator inputs (-). The amplitude of the perturbation inputs scale with the nominal actuator trajectory. The same perturbation inputs are used for the non-linear (- -) case and the cases with multiple linearizations (-.-) and a single linear model (---). The effect of the perturbation inputs can be appreciated in column 2 (for $\Delta \iota$ ) and column 3 (for $\Delta T_{\mathrm{e}}$ ) at several locations in the plasma.

The evolution of $\Delta T_{\mathrm{e}}$ using the multiple linearized models is in excellent agreement with the fully non-linear model for all shown locations. The evolution of $\Delta \iota$-profile shows a small difference in the centre of the plasma at $\rho=0, \rho=0.2$ and $\rho=0.4$. These differences are caused by the non-linear effect of the electron temperature $T_{\mathrm{e}}$ on the conductivity $\sigma_{\|}$, which influences the dynamics of the $\iota$-profile and cannot be contained accurately inside the multiple linearized state 
space models. While the quantitative estimates are not entirely correct for this region, the sign of the response is the same in both cases, which is essential for feedback control.

Prediction is in essence an open-loop simulation. For effective and reliable state constraint handling in MPC, prediction errors should be small, even at the end of the prediction horizon. Longer prediction horizons introduce increasing prediction errors due to model mismatches. Using the multiple linearizations results here in an error on the $\iota$-profile of less than 0.01 in magnitude.

The response of the single linear model shows that the $\Delta T_{\mathrm{e}}$ evolution is still in fair agreement with the non-linear model, except for the centre. However, a large deviation with respect to the non-linear case occurs in the $\Delta \iota$ evolution, especially at $0 \leqslant \rho \leqslant 0.6$. Note that the sign of the response is not correct at many times. Although the contribution of the bootstrap current in L-mode plasmas is small, the remaining non-linear terms (e.g. non-linear coupling of kinetic and magnetic profiles via plasma conductivity) prevent that a single linear model is not sufficient.

These specific results (not necessarily worst-case) indicate that the multiple linearized models describe accurately enough the dynamics in the broad vicinity of the nominal trajectory to justify the usage of these models in the MPC controller.

\subsection{Controller settings}

In this section we discuss the controller settings. The controller settings to be chosen are:

Reference. The nominal controlled variable evolution will function as a time-varying reference during the ramp-up phase, during the flat-top phase (after 120s) the reference is kept constant.

Prediction horizon $N$. The prediction horizon $N$ should be chosen such that the dominant effect of the actuators on the controlled variables is taken into account. From analysis of the eigenvalues of the system matrix $A_{k}$ that are dominant in the input-controlled variable behaviour (analysed via the socalled Hankel singular values [45]), we obtain that the slowest eigenmode in the flat-top of the nominal simulation has a timescale of $90 \mathrm{~s}$. This is the resistive diffusion time scale. We choose $N=78$ (equals $78 \mathrm{~s}$ ), being a compromise between on the one hand capturing crucial dynamics and on the other hand increasing prediction errors due to model uncertainties and higher computational cost.

Weights $Q, R_{\Delta U}$ and $W_{\varepsilon}$. In this work we obtained the settings of the weights $Q, R_{\Delta U}$ and $W_{\varepsilon}$ in the controller cost function by simulating the provided scenarios with varying these parameters around an initial intuitive guess and comparing robustness and performance. We noticed that especially the tight constraints on the plasma current (amplitude and ramp rate) and the available EC power in combination with a significant model mismatch in the electron heat transport reduced the clear meaning of the tuning parameters. However, using the fast simulator RAPTOR, it was possible to obtain a satisfying trade-off in a reasonable time.

The coefficients on the diagonal of $Q$ are chosen such that the error is only penalized in the region $\rho \leqslant 0.6 . \iota(\rho=1)$ (and hence the total current) is therefore free.
First the weight matrices are defined as a matrix having matrix norm equal to one multiplied by a scalar. By choosing $\frac{\left\|R_{\Delta U}\right\|_{2}}{10^{-12}}=1$ (normalizing for units in $\mathrm{W}$ or $\mathrm{A}$ ), we can define the scalar $W_{Q}=\|Q\|_{2}$. Varying $W_{Q}$ and $W_{\varepsilon}$ and simulating the provided scenarios led to the choice of $W_{Q}=5 \times 10^{3}$ and $W_{\varepsilon}=2 \times 10^{7}$.

Input parameterization $P_{\text {map }}$, including control horizon $N_{c}$. This is chosen as shown in figure 6 for all actuators. During the first six time instants every input is also a parameter, allowing for much control freedom in the beginning of the prediction horizon. Afterwards for each sixth time instant the input is a parameter up to the control horizon $N_{c}=36$ and the inputs in between are linearly interpolated. A spacing of six time instants allows still reasonable control freedom, while reducing the number of optimization parameters significantly. After the control horizon, the inputs are kept constant. Using this input parametrization reduces the number of free variables in the optimization from 396 to 61.

Coincidence time instants. The coincidence time instants are chosen as in figure 6. These overlap with the time instants at which the input parameters are defined, where additionally coincidence time instants are added after the control horizon $N_{c}=36$ at each sixth time instant. The number of time instants at which the states and controlled variables are predicted (and the state constraints are imposed) is this way reduced from 78 to 19 .

\section{Simulation results}

In this section we demonstrate the effectiveness of the MPC controller in simulations. First we illustrate tracking in the flat-top phase. Secondly we show handling of a sudden drop in available EC power by the MPC controller and finally we illustrate the tracking performance and constraint handling under plant-controller model mismatch caused by underestimated or overestimated thermal transport.

\subsection{Tracking the reference in flat-top phase}

In the first simulation case, tracking of the reference $\iota$-profile during the flat-top phase will be considered. The reference ı-profile at the beginning of the flat-top phase is not yet fully in stationary state, since the $U_{\mathrm{pl}}$-profile in figure 7 was not completely flat. Therefore the $\iota$-profile is expected to drift away from its reference in the absence of feedback control (only feedforward). The question is to what extent the feedback control will compensate for this drift. For this purpose, all feedforward actuator trajectories and prescribed profiles and quantities are extended from the end of the rampup until $400 \mathrm{~s}$. The reference $\iota$-profile during the flat-top phase is also taken at the end of the ramp-up phase.

The tracking error will be expressed by the normalized 2-norm of the vector of the error in the $\iota$-profile: $\| \iota-$ $\iota_{\text {ref }}\left\|_{2} /\right\| \iota_{\text {ref }} \|_{2}$. This error is only taken in the region $0 \leqslant \rho \leqslant$ 0.6 , consistent with the part of the $\iota$-profile which is weighted in the controller cost function.

The results for tracking the reference $\iota$-profile in the flattop phase are presented in figure 9 . In the feedforward only case (- -), the actuator commands (figures $9(a, e-h)$ ) are kept constant after the end of the ramp-up phase. This yields also 
- - - Nominal feedforward only ' - ' $\boldsymbol{-}$, With feedback
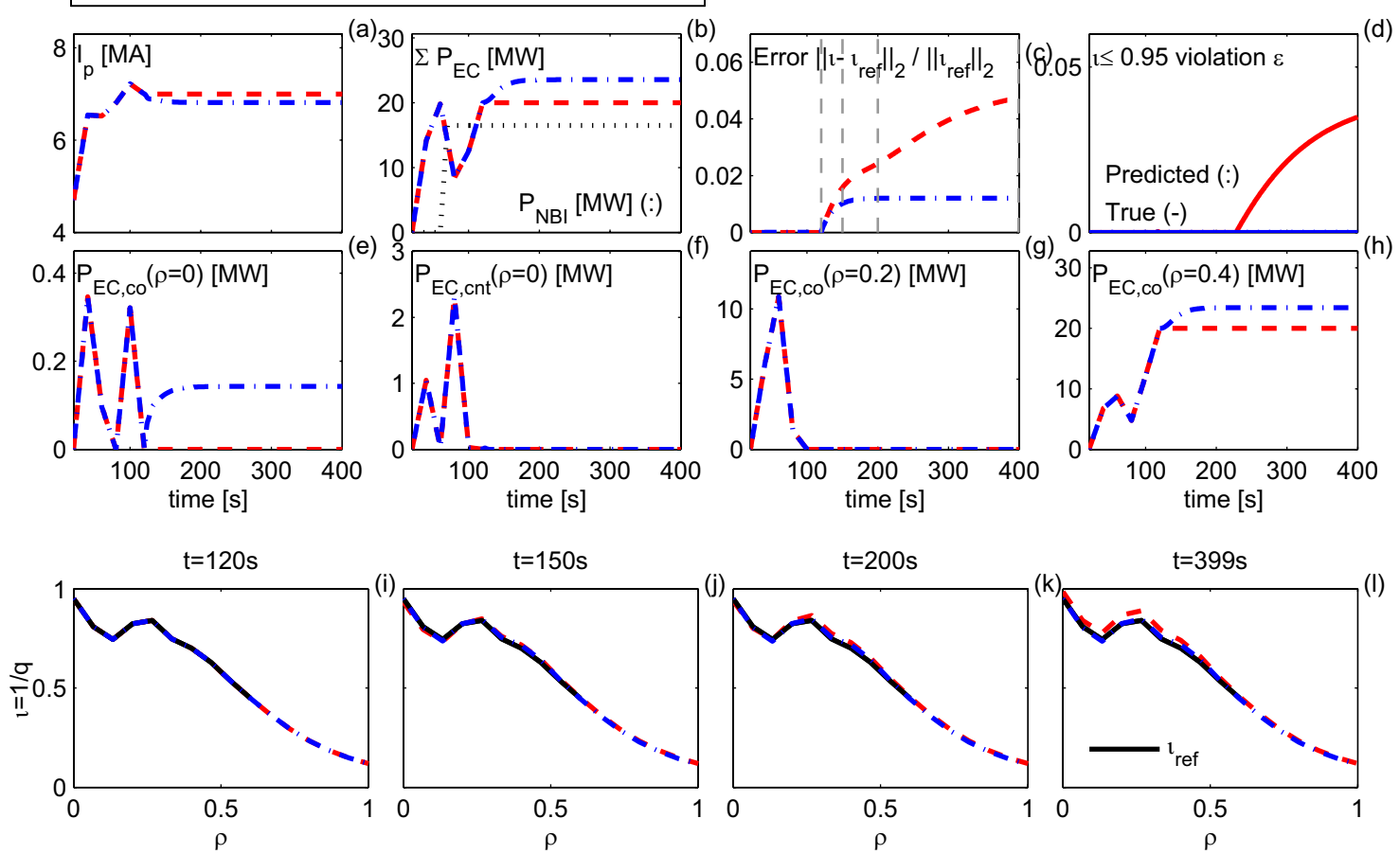

Figure 9. Tracking of reference $\iota$-profile in flat-top phase. Case with feedforward only (--) is shown together with feedback case (---). Top panels show time evolution of actuator commands of plasma current $I_{\mathrm{p}}(a)$, four EC beams $(e)-(h)$ and corresponding total EC-power $(b)$ error norm $(c)$ and soft constraint violation $(d)$. Bottom panels reveal $\iota$-profiles at time steps also indicated by grey dotted lines in top panels $(120,150,200$ and $399 \mathrm{~s})$. Reference $\iota$-profile is also shown for comparison $(-)$. Feedback controller reduces the tracking error $(c)$ and avoids the soft constraint violation $(d)$ by adjusting the actuators $(a),(e),(h)$.

a constant total EC power (figure $9(b)$ ). The resulting error norm (figure $9(c)$ ) increases as the $\iota$-profile drifts away from the reference $\iota$-profile and violates also the soft constraint to a large extent (figure $9(d)$ ). The resulting $\iota$-profile is given in the bottom panels (figures $9(i)-(l)$ ) for the time steps also indicated by the grey dotted lines in figure $9(c)$ : 120, 150, 200 and 399 s. In figure $9(l)$ can be observed clearly that the $\iota$-profile has evolved away from the reference (black) and has even entered the soft constraint region.

The feedback case (-.-) shows the performance of the MPC controller to track a reference -profile during the flat-top phase. The controller reduces the tracking error (figure 9(c)) significantly and avoids the soft constraint violation (figure $9(d)$ ) by lowering the plasma current $I_{\mathrm{p}}$ (figure $9(a)$ ) and adjusting the powers to the co-EC-beam at $\rho=0$ (figure $9(e)$ ) and the co-EC-beam at $\rho=0.4$ (figure $9(h)$ ). The reduction of the tracking error is also clearly shown in figure $9(l)$, where the $\iota$-profile is almost on top of its reference.

It can be concluded that the MPC controller can significantly reduce the error during tracking a fixed reference $\iota$-profile in the flat-top phase, despite the fact that the $\iota$-profile was not yet fully in stationary state at the start of the flat-top.

\subsection{Tracking with time-varying EC-power limit}

The next simulation case illustrates the handling of timevarying power limits. The goal is the same as in the previous case: tracking the reference $\iota$-profile during the flat-top phase. However, after $200 \mathrm{~s}$ we limit the maximum available EC power to $14 \mathrm{MW}$ instead of $25 \mathrm{MW}$. In figure 10 the response of the plasma to this limit will be compared between simply setting the available EC power to its maximum value (14 MW) and the case in which the MPC controller is aware of the timevarying limits.

The unconstrained EC-power case from figure 9 is repeated (-) and shown together with the saturated case (- -) and the case in which the MPC controller automatically takes the time-varying limits into account (---).

In the saturated case (- -), the actuator trajectories are taken from the unconstrained EC-power case and applied in open loop, where at $200 \mathrm{~s}$ the EC power to the beam at $\rho=0.4$ is reduced to $14 \mathrm{MW}$. In the absence of feedback, this results in an increasing error (figure $10(c)$ ) and a soft constraint violation exceeding early $\iota(\rho) \leqslant 1$ (figure $10(d)$ ). In figures $10(j)-(l)$, it noted that this violation happens at $\rho=0$ where $\iota(\rho)$ is largest.

In the case in which the MPC controller is aware of the time-varying limit (---), the controller aims at avoiding the soft constraint at the expense of a higher control error then without the strict limit $(c)$. At $200 \mathrm{~s}$, the maximum available EC power is indeed reduced to $14 \mathrm{MW}$ and the power to the EC beam at $\rho=0.4$ subsequently decreases further, the co-EC-beam at $\rho=0$ is shut off, while the EC power to the beam at $\rho=0.2$ is ramped up such that the maximum available EC power is used. This means an inward shift of the EC-power deposition. Notice the maximum ramp-down in the plasma current $I_{\mathrm{p}}$ immediately after $200 \mathrm{~s}$, after which the plasma current is kept at its minimum of $6 \mathrm{MA}$. The resulting change in the $\iota$-profile can be observed in figures 10(i)-(l). 

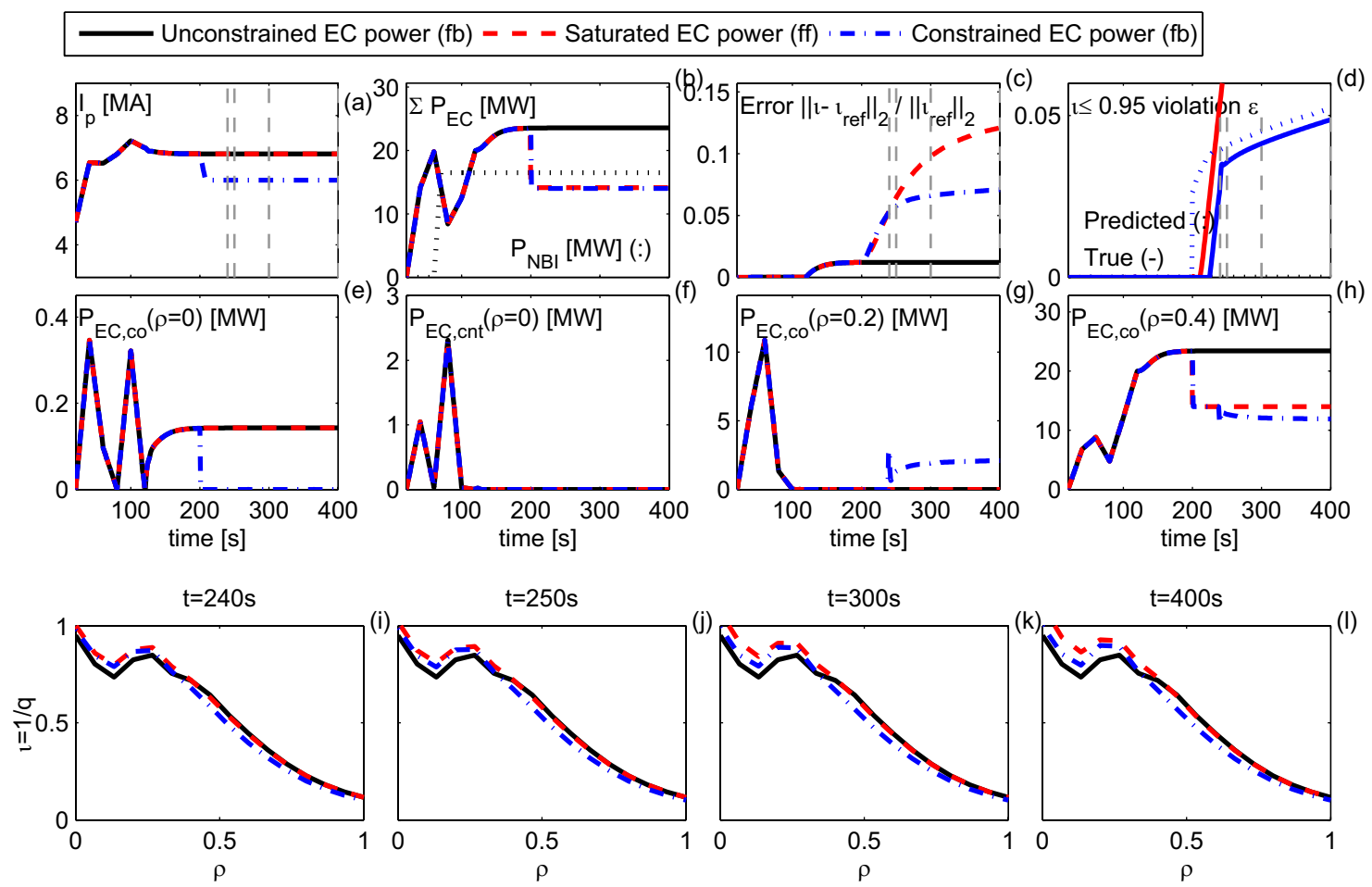

Figure 10. Saturation and handling of time-varying available EC-power constraint. The unconstrained feedback case from figure 9 is repeated (-). In the saturated case (- -), the actuator trajectories are copied from the unconstrained EC-power feedback case and applied in open loop, where the EC power to the beam at $\rho=0.4$ is reduced to $14 \mathrm{MW}$ after $200 \mathrm{~s}(h)$. This results in an increasing error $(c),(i)-(j)$ and soft constraint violation $(d)$. The MPC controller (---) limits the soft constraint violation $(d)$ at a smaller error norm then with manual saturation $(c)$, by adjusting the actuators $(a),(e),(g),(h)$, resulting in a different $\iota$-profile $(i)-(l)$.

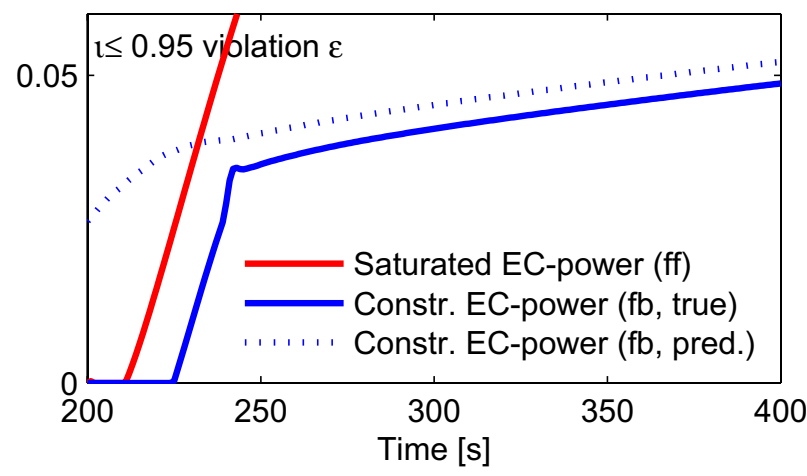

Figure 11. Detail of resulting soft constraint violation for saturation and automatic handling of time-varying available EC constraint. Note that already at $200 \mathrm{~s}$ a soft constraint violation is predicted to occur sometime within the prediction horizon. Note that the prediction is $35 \mathrm{~s}$ before the actual violation reaches the predicted point.

A detailed view of figure $10(d)$ in the time interval between 200 and $400 \mathrm{~s}$ is given in figure 11. Immediate after the available power drops, the controller predicts that the soft state constraint will be violated significantly within the prediction horizon. The actual soft state constraint violation reaches this point only $35 \mathrm{~s}$ later.

The prediction of the expected soft constraint violation together with the expected actuator and profile evolution can be provided to a supervisory controller. This would enable the supervisory controller to take adequate actions. In this case adequate actions may be (temporally) modifying the constraints on the $\iota$-profile and also modifying the reference $\iota$-profile towards a target which is achievable with the limited actuators. Alternatively, the supervisory controller can decide to switch to an entirely different plasma scenario depending on the experimental program in case of ITER. By those actions the controller would be able to compute desirable actuator inputs such that the plasma is maintained within the new limits.

\subsection{Tracking with model mismatches}

Model mismatches between the real system and the model in the controller are expected and the controller needs to compensate for them. Two simulation cases are therefore intended to show the performance of the MPC controller under a significant model mismatch during the ramp-up. In this example we change the transport model of the simulator model, while maintaining the same settings for the controller model as used in the previous cases.

4.3.1. Case with reduced thermal transport. In the first case with model mismatch, the empirical Bohm-gyroBohm transport coefficients are reduced by $30 \%$ with respect to the nominal case. This reduces the thermal transport significantly and hence higher temperatures (about $2 \mathrm{keV}$ difference in $T_{\mathrm{e}}(\rho=0)$ in flat-top) are achieved for the same heating power, affecting the conductivity profile and therefore also the current density evolution.

Figure 12 reveals the results. The reference (-) is shown for comparison. When these feedforward actuator trajectories are used in open loop (- -) in the simulator with model mismatch, 
- - - Model mismatch feedforward only ' - ' - ' Model mismatch with feedback
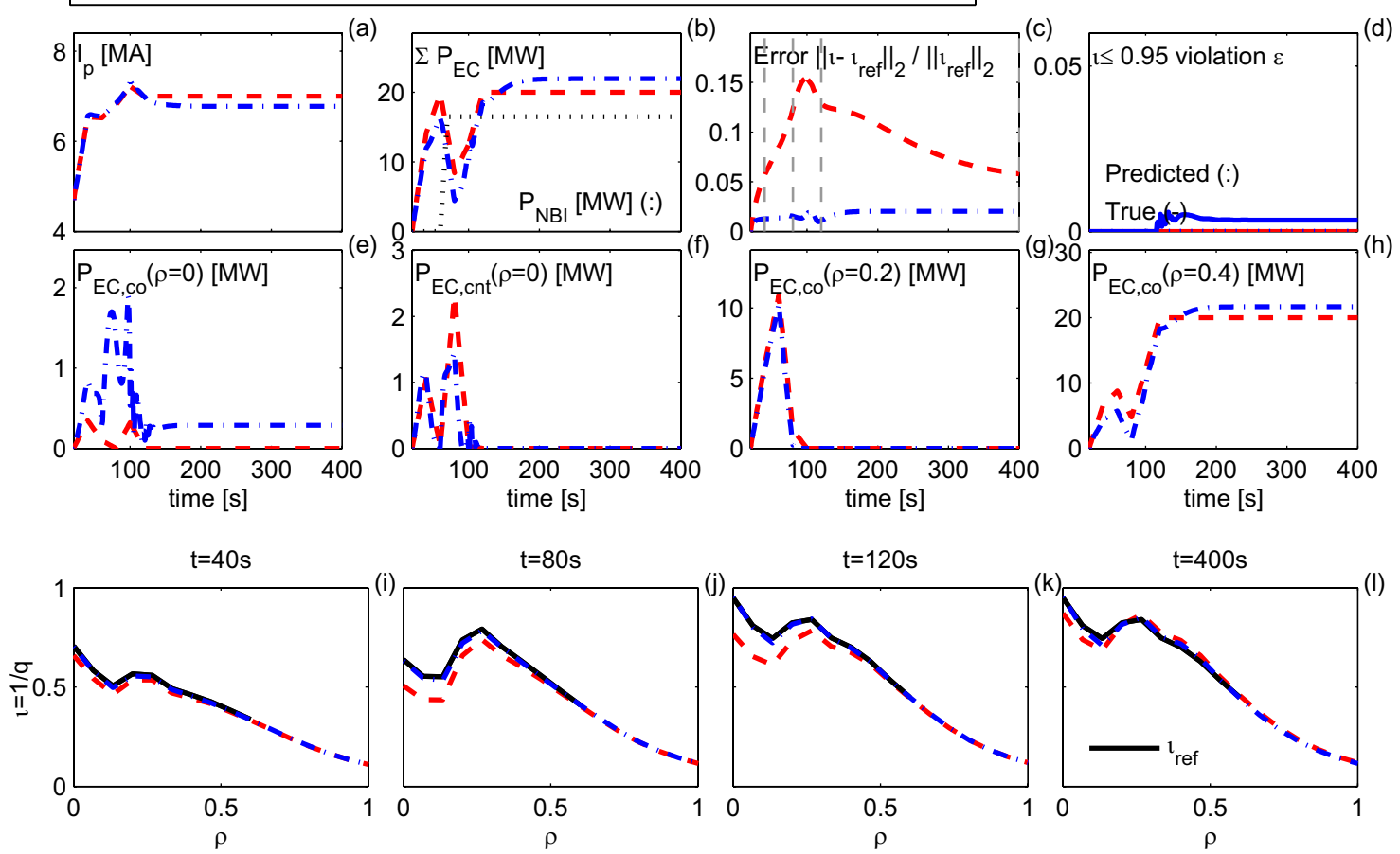

Figure 12. Tracking with decreased electron heat transport. The transport coefficients are reduced by $30 \%$ with respect to nominal case. Evolution subjected to model mismatch for feedforward only case (- -) is shown together with feedback controlled case (---). With only feedforward, the model mismatch results in a significant deviation from the reference $\iota$-profile $(c),(i)-(l)$, while $\iota$-profile slightly violates (unpredicted) the soft state constraint $(d)$. Feedback control reduces the tracking error significantly $(c)$, $(i)-(l)$, by adjusting the EC power to the several beams $(e)-(h)$ while $\iota$-profile slightly violates (unpredicted) the soft state constraint $(d)$.

this model mismatch causes a substantial deviation from the reference $\iota$-profile $(c),(i)-(l)$.

In the feedback controlled case (-.-) the tracking error can be significantly reduced by the MPC controller $(c),(i)-(l)$. The controller changes the actuator commands to the EC beams especially during the ramp-up $(e)-(h)$ at finally a slightly lower plasma current $I_{\mathrm{p}}(a)$.

The soft state constraint is slightly violated, but not predicted. This is caused by the error in the prediction model, leading to less accurate predictions and constraint handling. The fact that the EC power is still increased at reduced transport with respect to the feedforward case results in even higher (central) temperatures and hence even larger model mismatches in the current diffusion dynamics.

4.3.2. Case with increased thermal transport. In this second case with model mismatch the transport is increased, leading to a reduction of the central temperature of more than $1 \mathrm{keV}$ for the same heating power during the flat-top. We will show that this requires even more challenging control actions in which many actuator constraints become active.

Figure 13 reveals the results with increased thermal transport. Using only feedforward, results in a large error in the $\iota$-profile $(c),(i)-(l)$, while the $\iota$-profile also violates the soft state constraint $(d)$. This even results in $\iota(\rho)>1$ after $97 \mathrm{~s}$.

Feedback control avoids the soft state constraint violation (d) and reduces the error significantly during the ramp-up, whereas a significant error remains in the flat-top close to the error in the feedforward only case $(c)$. The controller pushes the actuators to the limits in ramp-rate $\left(I_{\mathrm{p}}, \mathrm{a}\right)$ and amplitude $(a, b)$, while distributing the EC power over the different beams $(e)-(h)$. A minor soft constraint violation is predicted, whereas a much smaller violation occurs temporally. This indicates again that the prediction is not fully accurate (as expected), due to the model mismatch.

Both simulation cases with model mismatch indicate that the MPC controller can reduce the tracking error due to a realistic model mismatch significantly, while handling the actuator and soft state constraints simultaneously. Taking the actuator limits actively into account allows for exploiting the full capabilities of the actuators.

\section{Discussion}

The performance is evaluated of an MPC controller for the control of the safety factor profile that actively takes the timevarying actuator and state constraints into account. In this section we discuss the results and provide directions to improve or extend this work. Implementing this MPC controller on an existing tokamak requires further validation in a more complete closed-loop simulation environment including plasma state reconstruction.

In the simulations we note a steady-state tracking error for which the current controller design cannot compensate. While a significant amount of the error is due to the fact that the reference profile is not achievable with the given set of model mismatches, disturbances and constraints, another part is because of the current design of the controller. The tracking error due to model mismatches and disturbances may be further reduced by including real-time disturbance estimations in the 


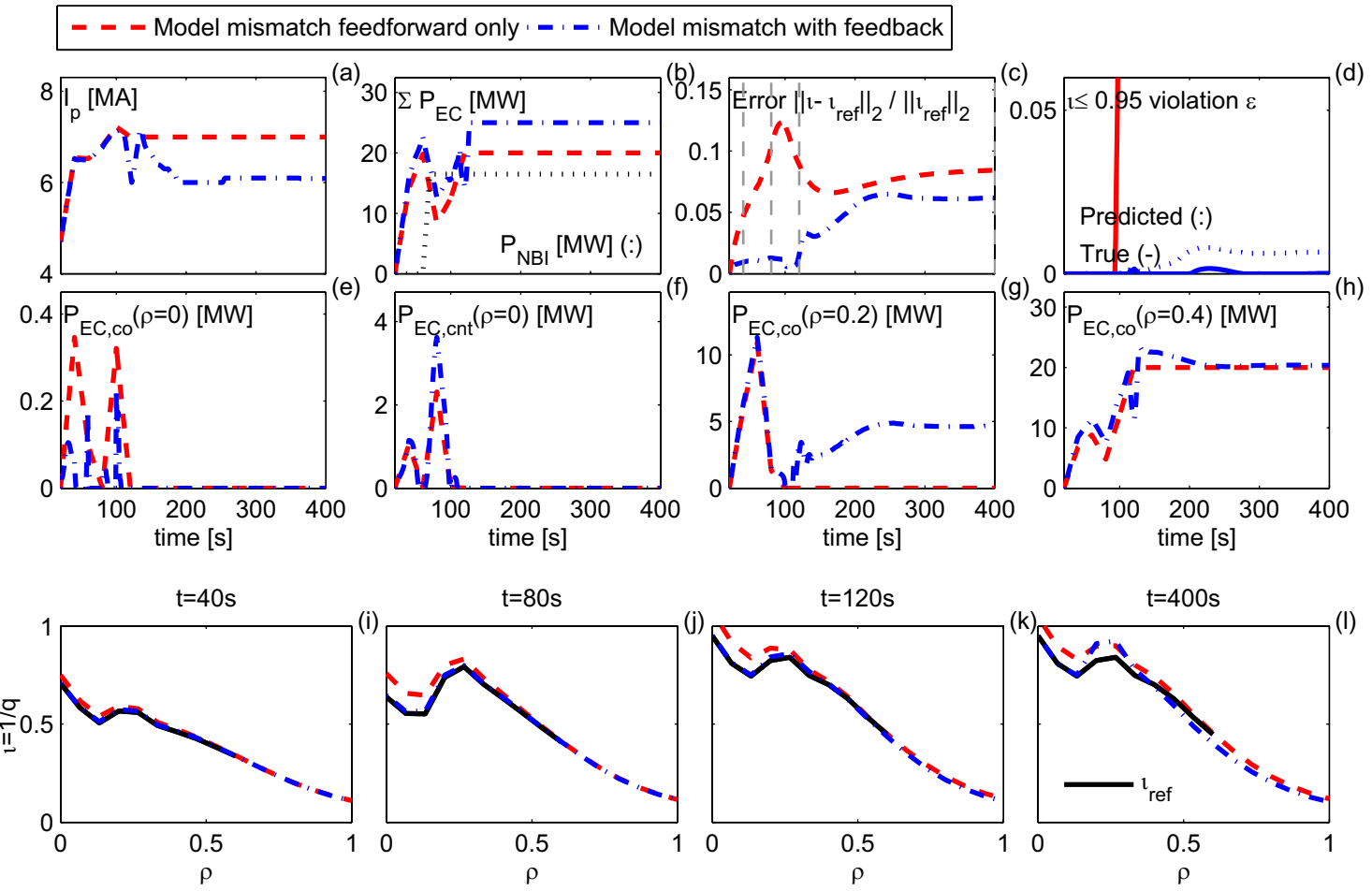

Figure 13. Tracking with increased electron heat transport. The transport coefficients are in this case increased by $30 \%$ with respect to nominal case. With only feedforward, the model mismatch results in a significant deviation from the reference $\iota$-profile $(c)$, $(i)-(l)$, while $\iota$-profile violates the soft state constraint such that even $\iota(\rho)>1$ after $97 \mathrm{~s}(d)$. Feedback control reduces the tracking error significantly during the ramp-up $(c),(i)-(l)$, by adjusting the EC power to the several beams $(e)-(h)$ and avoiding the soft state constraint violation. At the beginning of the flat-top, the EC power is increased to its maximum $(b)$ and distributed over the beams at $\rho=0.2(g)$ and $\rho=0.4(h)$, while the plasma current $I_{\mathrm{p}}(a)$ is decreased and increased at its ramp-rate limits and afterwards again decreased to close to its lower limit.

controller, a concept that is often used in MPC. This allows for (near) offset-free tracking performance in the flat-top phase and more accurate real-time profile evolution predictions. Disturbance estimations can be provided online, by using e.g. RAPTOR as observer [29].

The use of offline linearizations around a nominal trajectory limits excursions from these offline computed trajectories. However, the current design can be modified to include real-time varying references that are still within the vicinity of the offline computed nominal trajectory, but are more tractable depending on e.g. the real-time actuator constraints.

The controller does predict the true soft constraint violation only $35 \mathrm{~s}$ ahead instead of the prediction horizon of $78 \mathrm{~s}$. This indicates that the prediction is not fully accurate on those timescales (as mentioned before). Nevertheless, these $35 \mathrm{~s}$ warning time is already valuable information. The proposed extension of the controller for including disturbance estimates is expected to result in more accurate prediction of the soft constraint violations.

The MPC controller design (including disturbance estimates) is expected to satisfy the needs of profile control in case of normal well-prepared operation with well-behaved plasmas under modest uncertainty. Dealing with less well-behaved plasmas may be enabled by non-linear MPC approaches that are likely to be feasible on future larger scale tokamaks like ITER, having longer timescales of the profile evolution that provides more computational time. These nonlinear MPC approaches may allow for dealing with real-time varying references or control objectives, larger deviations from the nominal feedforward evolution and larger disturbances and constraint changes.

The flexibility of the proposed MPC controller to include time-varying constraints set by a supervisory controller is worth to be explored in future work, for instance for the simultaneous control of profiles and NTMs. The supervisory controller may use the real-time information of predictions of actuator and plasma state evolutions for allocating the shared actuator sources to the different control tasks, modifying references and state constraints and applying preemptive mitigation actions if disruptions cannot be avoided.

The required computational time can be further reduced by using faster dedicated algorithms and hardware to solve the online QP problem. This improvement may allow for implementation on faster time scales, e.g. smaller tokamaks or other control problems in fusion. One particular interesting case would be the simultaneous constraint handling for the plasma boundary control and the profile control. However, this requires the development of an integrated control-oriented model which solves self-consistently the non-linear coupled evolution of shape and profiles.

\section{Conclusions}

Active handling of time-varying actuator and plasma physics constraints is desired in the control of the safety factor profile in advanced operation of tokamaks. MPC is the only control method which can handle both time-varying actuator and 
state constraints. This work presented the design of an MPC controller and its application in closed-loop simulations.

The proposed MPC controller uses a prediction model, based on linearizations around a reference trajectory, to relate the future evolution of states and controlled variables to the present plasma state and future actuator commands. The MPC controller minimizes a cost function which penalizes the future error norm while avoiding too aggressive actuator inputs and violating soft state constraints. The minimization is subjected to time-varying actuator and plasma physics constraints. The resulting QP problem can be solved using dedicated solvers on a normal laptop within $8 \mathrm{~ms}$, which is fast enough to be implemented on currently operational tokamaks.

The potential of the MPC controller is demonstrated in closed-loop simulations using RAPTOR for an L-mode scenario with ITER parameters and a $q$-profile with $q>1$. Simulation examples show tracking of a reference $q$-profile evolution during the ramp-up and flat-top, even in cases of underestimated or overestimated transport in the transport model. Another simulation case reveals that the controller can handle a sudden reduction of the available EC power and in case that the constraints become too stringent, the MPC controller predicts that it cannot stay within the (soft) constraints in the near future. This allows one to provide a supervisory controller with warnings of these expected (soft) constraint violations and moreover with real-time predictions of the expected state and controlled variable evolution.

Finally, it is discussed that the MPC controller can be extended and improved to allow for (near) offset-free tracking in the flat-top phase and more accurate real-time profile evolution predictions and hereby more reliable state constraint handling. The MPC controller is proposed to allow for dynamic actuator sharing for the simultaneous control of profiles and NTMs. Reduction of the required computational time may open up new applications in fusion.

\section{Acknowledgments}

The first author gratefully acknowledges the discussions with Hugo van den Brand and Jonathan Citrin of FOM DIFFER and Olivier Sauter of CRPP-EPFL. This work has been performed in the framework of the NWO-RFBR Center of Excellence (grant 047.018.002) on Fusion Physics and Technology. This work is supported in part by the Netherlands Organization for Scientific Research and the European Communities. It was carried out within the framework of the European Fusion Programme. The views and opinions expressed herein do not necessarily reflect those of the European Commission.

\section{Appendix A. Definitions and derivations}

In this appendix we provide the definitions and derivations as used in section 2 .

\section{A.1. Prediction matrices}

For the use in the prediction equations (7), the following notation is introduced:

$$
\coprod_{l=m}^{n} A_{k+l} \equiv\left\{\begin{array}{ccc}
A_{k+n} A_{k+n-1} \ldots A_{k+m} & \text { if } & m \leqslant n \\
I & \text { if } & m>n
\end{array}\right.
$$

The prediction matrices $\Gamma_{A_{k, N}}, \Gamma_{B_{k, N}}, \Gamma_{C_{k, N}}$ and $\Gamma_{D_{k, N}}$ can now be written as follows:

$\Gamma_{A_{k, N}}=\left[\begin{array}{c}\coprod_{l=1}^{0} A_{k+l} \\ \coprod_{l=1}^{1} A_{k+l} \\ \vdots \\ \coprod_{l=1}^{N-1} A_{k+l}\end{array}\right] A_{k}$,

$\Gamma_{B_{k, N}}=\left[\begin{array}{ccccc}{\left[\bigcup_{l=1}^{0} A_{k+l}\right] B_{k}} & 0 & \cdots & 0 & 0 \\ {\left[\bigcup_{l=1}^{1} A_{k+l}\right] B_{k}} & {\left[\coprod_{l=2}^{1} A_{k+l}\right] B_{k+1}} & \ddots & \ddots & \vdots \\ \vdots & \vdots & \ddots & 0 & 0 \\ {\left[\bigcup_{l=1}^{N-1} A_{k+l}\right] B_{k}} & {\left[\coprod_{l=2}^{N-1} A_{k+l}\right] B_{k+1}} & \cdots & {\left[\coprod_{l=N}^{N-1} A_{k+l}\right] B_{k+N-1}} & 0\end{array}\right]$,

$\Gamma_{C_{k, N}}=\left[\begin{array}{c}C_{k+1} \coprod_{l=1}^{0} A_{k+l} \\ 1 \\ C_{k+2} \coprod_{l=1} A_{k+l} \\ \vdots \\ C_{k+N} \coprod_{l=1}^{N-1} A_{k+l}\end{array}\right] A_{k}$

and

$\Gamma_{D_{k, N}}=$
$\left[\begin{array}{ccccc}C_{k+1}\left[\bigcup_{l=1}^{0} A_{k+l}\right] B_{k} & D_{k+1} & \cdots & 0 & 0 \\ C_{k+2}\left[\bigcup_{l=1}^{1} A_{k+l}\right] B_{k} & C_{k+2}\left[\bigcup_{l=2}^{1} A_{k+l}\right] B_{k+1} & D_{k+2} & \ddots & \vdots \\ \vdots & \vdots & \ddots & \ddots & \\ C_{k+N}\left[\bigcup_{l=1}^{N-1} A_{k+l}\right] B_{k} & C_{k+N}\left[\bigcup_{l=2}^{N-1} A_{k+1}\right] B_{k+1} & \cdots & C_{k+N}\left[\bigcup_{l=N}^{N-1} A_{k+l}\right] B_{k+N-1} & D_{k+N}\end{array}\right]$.

\section{A.2. Constraints}

Here we derive the linear inequality constraints (8) and (9) representing the actuator and state constraints.

A.2.1. Actuator constraints. The actuator amplitude constraints are given as

$$
U_{\min , k} \leqslant U_{k, N} \leqslant U_{\max , k}
$$

Using (6) these constraints can be rewritten as

$$
\left[\begin{array}{c}
I \\
-I
\end{array}\right] \tilde{U}_{k, N} \leqslant\left[\begin{array}{c}
U_{\max , k}-U_{k, N}^{0} \\
-U_{\min , k}+U_{k, N}^{0}
\end{array}\right] .
$$

Following (11), actuator ramp-rate constraints are given as

$$
\Delta U_{\min , k} \leqslant \Delta U_{k, N} \leqslant \Delta U_{\max , k} .
$$

Using (6) and (11) these constraints can be rewritten as

$$
\left[\begin{array}{c}
\Gamma_{\Delta} \\
-\Gamma_{\Delta}
\end{array}\right] \tilde{U}_{k, N} \leqslant\left[\begin{array}{c}
\Delta U_{\max , k}-\Gamma_{\Delta} U_{k, N}^{0} \\
-\Delta U_{\min , k}+\Gamma_{\Delta} U_{k, N}^{0}
\end{array}\right] .
$$


The mixed actuator constraints are given as

$$
A_{U, \text { mix }} U_{k, N} \leqslant b_{U, \text { mix }}
$$

Using (6) these constraints can be rewritten as

$$
A_{U, \text { mix }} \tilde{U}_{k, N} \leqslant b_{U, \text { mix }}-A_{U, \text { mix }} U_{k, N}^{0} .
$$

Combining now the equations (A.7), (A.9) and (A.11) we obtain

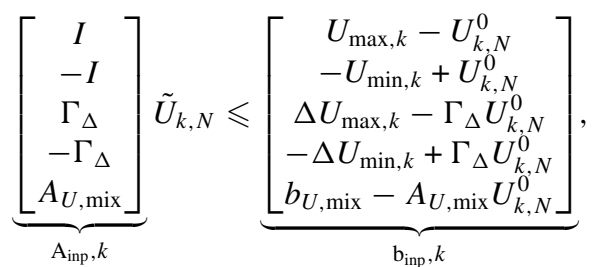

which defines the matrices as given in (8).

A.2.2. State constraints State constraints are defined as

$$
A_{X, \text { mix }} X_{k+1, N} \leqslant b_{X, \text { mix }}+\varepsilon I \text {. }
$$

Rewriting (A.13) using (6) and (7) results in

$$
\begin{aligned}
& \underbrace{\left[A_{X, \text { mix }} \Gamma_{B_{k, N}}-I\right]}_{A, \text { state }, k}\left[\begin{array}{c}
\tilde{U}_{k, N} \\
\varepsilon
\end{array}\right] \\
& \leqslant \underbrace{\left[b_{X, \text { mix }}-A_{X, \text { mix }}\left[X_{k+1, N}^{0}+\Gamma_{A_{k, N}} \tilde{x}_{k}\right]\right]}_{b_{\text {state }, k}},
\end{aligned}
$$

which defines the matrices as given in (9).

\section{A.3. Cost function}

The cost function $J_{k}$ as given in (10) can be rewritten by using (7) and (11):

$$
\begin{aligned}
J_{k}= & \tilde{x}_{k}^{T} \Gamma_{C_{k, N}}^{T} Q \Gamma_{C_{k, N}} \tilde{x}_{k} \\
& +\tilde{x}_{k}^{T}\left[2 \Gamma_{C_{k, N}}^{T} Q \Gamma_{D_{k, N}}\right] \tilde{U}_{k, N} \\
& +\tilde{U}_{k, N}^{T}\left[\Gamma_{D_{k, N}}^{T} Q \Gamma_{D_{k, N}}+R_{\Delta \tilde{U}}\right] \tilde{U}_{k, N} \\
& +w_{\varepsilon} \varepsilon^{2}
\end{aligned}
$$

In the minimization of $J_{k}$ with respect to the future input sequence $\tilde{U}_{k, N}$, the constant part depending not on $\tilde{U}_{k, N}$ can be neglected. The reduced cost function $\hat{J}_{k}$ reads then as follows:

$$
\begin{aligned}
\hat{J}_{k}=\tilde{x}_{k}^{T} & \underbrace{\left[2 \Gamma_{C_{k, N}}^{T} Q \Gamma_{D_{k, N}}\right]}_{\Gamma_{J_{1}, k}} \tilde{U}_{k, N} \\
& +\tilde{U}_{k, N}^{T} \underbrace{\left[\Gamma_{D_{k, N}}^{T} Q \Gamma_{D_{k, N}}+R_{\Delta \tilde{U}}\right]}_{\Gamma_{J_{2}, k}} \tilde{U}_{k, N}+w_{\varepsilon} \varepsilon^{2} .
\end{aligned}
$$

\section{A.4. QP matrices and vectors}

By implementing the input vector parametrization in (12) and using $\xi_{k}^{T}=\left[\begin{array}{ll}\tilde{p}_{k, N}^{T} & \varepsilon\end{array}\right]$, then the cost function (A.16), actuator constraints (A.12) and state constraints (A.14) can be rewritten into the standard form of the QP problem (13) at each time instant $t_{k}$ :

$\hat{J}_{k}=\tilde{x}_{k}^{T} \underbrace{\left[\begin{array}{ll}\Gamma_{J_{1}, k} P_{\text {map }} & 0\end{array}\right]}_{F_{k}} \xi_{k}+\xi_{k}^{T} \underbrace{\left[\begin{array}{cc}P_{\text {map }}^{T} \Gamma_{J_{2}, k} P_{\text {map }} & 0 \\ 0 & w_{\varepsilon}\end{array}\right]}_{2 H_{k}} \xi_{k}$,

$\underbrace{\left[\begin{array}{cc}A_{\text {inp }} & 0 \\ A_{\text {state }}\end{array}\right]\left[\begin{array}{cc}P_{\text {map }} & 0 \\ 0 & 1\end{array}\right]}_{A_{\text {ineq }, k}} \xi_{k} \leqslant \underbrace{\left[\begin{array}{c}b_{\text {inp }, k} \\ b_{\text {state }, k}\end{array}\right]}_{b_{\text {ineq }, k}}$,

$\underbrace{\left[\begin{array}{ll}I_{n_{u}} & 0\end{array}\right]\left[\begin{array}{ll}P_{\text {map }} & 0\end{array}\right]}_{A_{\text {eq }}} \xi_{k}=\underbrace{\left[\tilde{u}_{k}\right.}_{b_{\text {eq }, k}}]$,

which defines the matrices $F_{k}, H_{k}, A_{\text {ineq }, k}$ and $A_{\text {eq }}$ and the vectors $b_{\text {ineq, } k}$ and $b_{\text {eq }, k}$ in (13).

\section{References}

[1] Gribov Y. et al 2007 Nucl. Fusion 47 S385

[2] Snipes J. et al 2014 Fusion Eng. Des. 89507

[3] Ishida S. et al 2011 Nucl. Fusion 51094018

[4] Grosman A. et al 2013 Fusion Eng. Des. 88497

[5] Ou Y. et al 2008 Plasma Phys. Control. Fusion 50115001

[6] Xu C. et al 2010 IEEE Trans. Plasma Sci. 38163

[7] Felici F. et al 2012 Plasma Phys. Control. Fusion 54025002

[8] Moreau D. et al 2013 Nucl. Fusion 53063020

[9] Witrant E. et al 2007 Plasma Phys. Control. Fusion 491075

[10] Ou Y. et al 2007 Fusion Eng. Des. 821153

[11] Felici F. et al 2011 Nucl. Fusion 51083052

[12] Gaye O. et al 2013 Control. Eng. Pract. 21 1350-8

[13] Ou Y. et al 2011 Control Eng. Pract. 1922

[14] Ouarit H. et al 2011 Fusion Eng. Des. 861018

[15] Xu C. et al 2011 Automatica 47418

[16] Bribiesca Argomedo F. et al 2013 Nucl. Fusion 53033005

[17] Barton J.E. et al 2012 Nucl. Fusion 52123018

[18] Boyer M.D. et al 2012 American Control Conf. (ACC) (2012) pp 2996-3001

[19] Kim S. et al 2012 Nucl. Fusion 52074002

[20] Boyer M.D. et al 2013 Plasma Phys. Control. Fusion $\mathbf{5 5} 105007$

[21] Gaye O. et al 2013 Automatica 492795

[22] Vu N.T.M. et al 2013 Decision and Control (CDC) (2013 IEEE 52nd Annual Conference) pp 4176-81

[23] Winter A. et al 2014 Fusion Eng. Des. 89 267-72

[24] Snipes J. et al 2010 Fusion Eng. Des. 85461

[25] Kamada Y. et al 2013 Nucl. Fusion 53104010

[26] Camacho E. et al 2004 Model Predictive Control (London: Springer) ISBN 9781852336943

[27] Maciejowski J. 2002 Predictive Control with Constraints (Englewood Cliffs, NJ: Prentice-Hall)

[28] Rossiter J. 2013 Model-Based Predictive Control: A Practical Approach (London: Taylor and Francis) ISBN 9780203503966

[29] Felici F. et al 2012 Proc. 24th Int. Conf. on Fusion Energy (San Diego, CA, 2012) EX/P3-12 www-naweb.iaea.org/ napc/physics/FEC/FEC2012/papers/169_EXP312.pdf

[30] Hinton F.L. et al 1976 Rev. Mod. Phys. 48239

[31] Sauter O. et al 1999 Phys. Plasmas 6 2834-9

[32] Sauter O. et al 2002 Phys. Plasmas 95140

[33] Erba M. et al 1998 Nucl. Fusion 381013

[34] van Dongen J. et al 2015 Numerical optimization of actuator trajectories for ITER hybrid scenario profile evolution Plasma Phys. Control. Fusion at press

[35] Artaud J. et al 2010 Nucl. Fusion 50043001

[36] Hogeweij G. et al 2013 Nucl. Fusion 53013008

[37] Grüne L. et al 2011 Nonlinear Model Predictive Control: Theory and Algorithms (Berlin: Springer) 
[38] Hespanha J.P. 2009 Linear Systems Theory (Princeton, NJ: Princeton University Press)

[39] Grimble M.J. et al 2014 Nonlinear Industrial Control Systems: Design and Applications (London: Springer)

[40] Richalet J. et al 2009 Predictive Functional Control (Berlin: Springer) ISBN 9781848824935

[41] Wills A. 2007 Quadratic Programming in C Revision 2007 http://sigpromu.org/quadprog/
[42] Ferreau H.J. et al 2008 Int. J. Robust Nonlinear Control 18816

[43] Hobirk J. et al 2012 Plasma Phys. Control. Fusion 54095001

[44] Ljung L. 1998 System Identification: Theory for the User (Essex: Pearson Education)

[45] Skogestad S. et al 2005 Multivariable feedback control: Analysis and Design (New York: Wiley) ISBN 9780470011676 